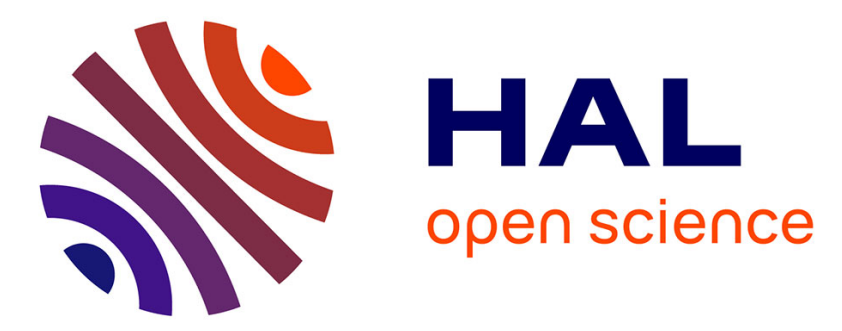

\title{
Uncertainty principles and characterization of the heat kernel for certain differential-reflection operators
}

\author{
Salem Ben Saïd, Asma Boussen, Mohamed Sifi
}

\section{To cite this version:}

Salem Ben Saïd, Asma Boussen, Mohamed Sifi. Uncertainty principles and characterization of the heat kernel for certain differential-reflection operators. Advances in Pure and Applied Mathematics, 2015, 6 (4), pp.215-239. 10.1515/apam-2015-5010 . hal-01282492

\section{HAL Id: hal-01282492 \\ https://hal.science/hal-01282492}

Submitted on 3 Mar 2016

HAL is a multi-disciplinary open access archive for the deposit and dissemination of scientific research documents, whether they are published or not. The documents may come from teaching and research institutions in France or abroad, or from public or private research centers.
L'archive ouverte pluridisciplinaire HAL, est destinée au dépôt et à la diffusion de documents scientifiques de niveau recherche, publiés ou non, émanant des établissements d'enseignement et de recherche français ou étrangers, des laboratoires publics ou privés. 


\title{
UNCERTAINTY PRINCIPLES AND CHARACTERIZATION OF THE HEAT KERNEL FOR CERTAIN DIFFERENTIAL-REFLECTION OPERATORS
}

\author{
SALEM BEN SAID, ASMA BOUSSEN \& MOHAMED SIFI
}

\begin{abstract}
We prove various versions of uncertainty principles for a certain Fourier transform $\mathscr{F}_{A}$. Here $A$ is a Chébli function (i.e. a Sturm-Liouville function with additional hypotheses).

We mainly establish an analogue of Beurling's theorem, and its relatives such as theorems of type Gelfand-Shilov, Morgan's, Hardy's, and Cowling-Price, for $\mathscr{F}_{A}$, and relating them to the characterization of the heat kernel corresponding to $\mathscr{F}_{A}$.

Heisenberg's and local uncertainty inequalities were also proved.
\end{abstract}

\section{INTRODUCTION}

One of the most famous and paradoxical predictions of quantum theory is the statement that the position and the velocity of an object cannot both be measured exactly at the same time, even in theory. The quantitative version of this phenomenon is the Heisenberg uncertainty principle, which may be formulated as an inequality in Fourier analysis [38]. Roughly speaking, Heisenberg's inequality asserts that a nonzero function $f$ and its Euclidean Fourier transform $\hat{f}$ cannot both be very small.

The uncertainty principle has also an interpretation in classical physics. Suppose $f(t)$ is a sound wave at time $t$, and denote by $\operatorname{Var}(f)$ an index of its concentration in time. We interpret $\hat{f}(t)$ as the frequency distribution of the sound wave at time $t$, so $\operatorname{Var}(\hat{f})$ is an index of the concentration of the pitch. The uncertainty principle then says that a sound cannot be very concentrated in both time and pitch. In particular, short duration tones will have a poorly determined pitch. Singers take advantage of this: in very rapid passages, errors in intonation will not be noticeable.

Heisenberg's uncertainty principle tells us that if $f$ is highly localized, then $\hat{f}$ cannot be concentrated near a single point, but it does not say anything about $\hat{f}$ being concentrated in a small neighborhood or perhaps a finite number of widely separated points. In fact, the latter phenomenon cannot occur either, and it is the object of local uncertainty inequalities to make this precise. The first such inequalities were obtained by Faris [14], and they were subsequently sharpened and generalized by Price [29, 30]. See also [28] and [23].

Heisenberg's inequality is an instance of various quantitative versions of uncertainty principles due to Hardy in 1933 [18], Morgan in 1934 [25], Gelfand-Shilov in 1953 [17], Cowling-Price in 1983 [12], etc. Further details on uncertainty principles can be

2000 Mathematics Subject Classification. 42A38, 26D15, 33C47.

Keywords. Differential-reflection operators, heat kernel, uncertainty principles. 
found in the textbook of Havin and Joricke [19], and in the expository papers of Havin [20] and Folland-Sitaram [15].

Building on the paper [18] by Hardy, around 1964 Beurling proved a new version of Heisenberg's uncertainty principle, which found a lot of success for its elegance and simplicity. This result appeared without proof in the collected works [3] of Beurling. In 1991, Hörmander [22] reproduced a proof from the notes he made when Beurling explained this result to him during a private conversation. It is known by now that Beurling's uncertainty principle is the master theorem of this subject in the sense that it implies the theorems of Hardy, Morgan, Gelfand-Shilov, and Cowling-Price. Recently, this theorem was further generalized by Bonami, Demange, and Jaming [5]. Analogues of Beurling's theorem in the context of Lie groups have been studied in [32, 2, 27, 33, 31] etc.

In this paper our aim is to prove the analogue of the above mentioned uncertainty principles for a generalized Fourier transform associated with a wide family of differentialreflection operators.

More precisely, we consider the operator

$$
\Lambda_{A} f(x)=f^{\prime}(x)+\frac{A^{\prime}(x)}{A(x)}\left(\frac{f(x)-f(-x)}{2}\right),
$$

where $A$ is so-called a Chébli function on $\mathbb{R}$ (i.e. a Sturm-Liouville function with additional hypotheses). In particular, the function $A$ assumes:

$$
A(x)=|x|^{2 \alpha+1} B(x)
$$

where $\alpha>-\frac{1}{2}$ and $B$ is any even, positive and smooth function with $B(0)=1$, and the limit

$$
2 \varrho:=\lim _{x \rightarrow+\infty} \frac{A^{\prime}(x)}{A(x)}
$$

exists and nonnegative.

For instance, if $A(x)=|\sinh x|^{2 \alpha+1}(\cosh x)^{2 \beta+1}$, with $\alpha>\beta \geq-1 / 2$, then the operator (1.1) reduces to Heckman's operator in one dimension [21]. Operators of type (1.1) have a long history going back to the pioneering paper [13] by Dunkl.

For $\lambda \in \mathbb{C}$, denote by $\Psi(\lambda, x)$ the unique solution to the equation $\Lambda_{A} f(x)=i \lambda f(x)$ with the initial data $f(0)=1$. In particular, for $\lambda, x \in \mathbb{R}$ we have $|\Psi(\lambda, x)| \leq 1$.

On the space $L^{1}(\mathbb{R}, A(x) d x)$ we consider the Fourier transform $\mathscr{F}_{A}$ defined by

$$
\mathscr{F}_{A} f(\lambda)=\int_{\mathbb{R}} f(x) \Psi(\lambda,-x) A(x) d x, \quad \lambda \in \mathbb{R} .
$$

By now, the transform $\mathscr{F}_{A}$ is very well understood. See [4] for a detailed harmonic analysis related to $\mathscr{F}_{A}$.

For $t>0$ and $x \in \mathbb{R}$, let $h_{t}(x)=h(t, x):=\mathscr{F}_{A}^{-1}\left(e^{-t \lambda^{2}}\right)(x)$ be the heat kernel associated with the heat semigroup $e^{t \Lambda_{A}^{2}}$. In particular, the map $x \mapsto h(t, x)$ is even and belongs to the space $L^{1}(\mathbb{R}, A(x) d x)$. The estimates proved in [16] for $h(t, x)$ will play a crucial rule in several places. 
Below we will write $\|\cdot\|_{L_{x}^{p}}$ and $\|\cdot\|_{L_{\lambda}^{p}}$ instead of $\|\cdot\|_{L^{p}(\mathbb{R}, A(x) d x)}$ and $\|\cdot\|_{L^{p}(\mathbb{R}, \xi(\lambda) d \lambda)}$, respectively. Here $\xi(\lambda) d \lambda$ denotes a Plancherel measure supported on $\mathbb{R} \backslash]-\varrho, \varrho[$, which will be given explicitly in the next section, where $\varrho$ is as in (1.3).

Define the following domain

$$
\mathbb{D}_{\varrho}:= \begin{cases}\{1\} & \text { if } \varrho=0, \\ \left(\frac{1}{2}, 1\right] & \text { if } 0<\varrho<1, \\ {\left[\frac{1}{2}, 1\right]} & \text { otherwise. }\end{cases}
$$

The Heisenberg inequality for $\mathscr{F}_{A}$ reads:

Theorem A (Heisenberg's type; see Theorem 3.3). Let $r$, s be two positive constants and $\gamma \in \mathbb{D}_{\varrho}$. Then, for all $f \in L^{2}(\mathbb{R}, A(x) d x)$ we have

$$
\left\||x|^{\gamma s} f\right\|_{L_{x}^{2}}^{\frac{r}{r+s}}\left\||\lambda|^{r} \mathscr{F}_{A}(f)\right\|_{L_{\lambda}^{2}}^{\frac{s}{r+s}} \gtrsim\|f\|_{L_{x}^{2}}
$$

The proof of this theorem uses a similar approach to that employed in [8] for a subLaplacian on a Lie group of polynomial volume growth.

As mentioned earlier, the local uncertainty principle comes to fill in the blank left by Heisenberg's uncertainty principle. For $\mathscr{F}_{A}$, the local uncertainty inequality states:

Theorem B (the local type; see Theorem 4.2). Let $s$ be a positive constant so that $s<\alpha+1$, and let $E \subseteq \mathbb{R} \backslash]-\varrho, \varrho[$ be a measurable set such that $0<\xi(E)<\infty$. Then, for all nonzero function $f \in L^{2}(\mathbb{R}, A(x) d x)$, we have

$$
\left\|f *_{A} w_{E}\right\|_{L_{x}^{2}} \supsetneqq c_{s, E}\left\|w_{E}\right\|_{L_{x}^{2}}^{\frac{s}{\alpha+1}}\left\||x|^{s} f\right\|_{L_{x}^{2}},
$$

where $w_{E}:=\mathscr{F}_{A}^{-1} \chi_{E} \in L^{2}(\mathbb{R}, A(x) d x)$.

We now turn our attention to an analogue of Beurling's uncertainty principle, which has been extended to many directions and contexts. The principle result of this paper is to prove Beurling's theorem, and its relatives such as the theorems of Gelfand-Shilov and Cowling-Price, for the Fourier transform $\mathscr{F}_{A}$.

Theorem C (Beurling's type; see Theorem 5.1). Let $f \in L^{2}(\mathbb{R}, A(x) d x)$ satisfy

$$
\int_{\mathbb{R}} \int_{\mathbb{R}} \frac{|f(x)|\left|\mathscr{F}_{A}(f)(\lambda)\right| e^{|x||\lambda|}}{(1+|x|+|\lambda|)^{N}} A(x) d x \xi(\lambda) d \lambda<\infty,
$$

for some nonnegative integer $N$. Then $f$ is of the form

$$
f(x)=\sum_{k=0}^{n} c_{k} \Lambda_{A, x}^{(k)} h(t, x) \quad n<\frac{N-(2 \alpha+2)}{2},
$$

where $c_{k}$ are complex constants, and the subscript $x$ in $\Lambda_{A, x}^{(k)} h(t, x)$ denotes the relevant variable in the $k$-th power of the operator $\Lambda_{A}$ applied to the heat kernel $h(t, x)$ for some $t>0$. 
In the limit case $\alpha=-1 / 2$, with $B(x)=1$ for all $x \in \mathbb{R}$, the above statement collapses to the Bonami-Demange-Jaming result in one dimension [5].

Theorem $\mathrm{C}$ has several interesting consequences. We begin with the following variation of the uncertainty principle involving conjugate exponents $p$ and $q$ :

Theorem D (Gelfand-Shilov type; see Theorem 6.1). Let $f \in L^{2}(\mathbb{R}, A(x) d x)$ and $N$ be a nonnegative integer. Assume that

$$
\int_{\mathbb{R}} \frac{|f(x)| e^{\frac{(2 s) p}{p}|x|^{p}}}{(1+|x|)^{N}} A(x) d x<\infty, \quad \int_{\mathbb{R}} \frac{\left|\mathscr{F}_{A}(f)(\lambda)\right| e^{\frac{(2 t)^{q}}{q}|\lambda|^{q}}}{(1+|\lambda|)^{N}} \xi(\lambda) d \lambda<\infty,
$$

where $1<p<\infty, \frac{1}{p}+\frac{1}{q}=1$, and $s$, $t$ are positive constants.

1) If $s t>\frac{1}{4}$ then $f=0$ almost everywhere.

2) If $s t=\frac{1}{4}$ and $p \neq 2$ (hence $q \neq 2$ ) then $f=0$ almost everywhere.

3) If $s t=\frac{1}{4}$ and $p=q=2$, then

$$
f(x)=\sum_{k=0}^{n} c_{k} \Lambda_{A, x}^{(k)} h\left(2 t^{2}, x\right) \quad n<N-(2 \alpha+2) .
$$

In particular, if $2 \alpha+2<N \leq 2 \alpha+3$ then $f(x)=c_{0} h\left(2 t^{2}, x\right)$, where $c_{0}$ is an arbitrary constant for $\varrho=0$, and $c_{0}=0$ for $\varrho>0$.

The uncertainty principle below follows partially from Theorem $\mathrm{D}$ above.

Theorem E (Morgan's type; see Theorem 6.2). Suppose a measurable function $f: \mathbb{R} \rightarrow$ C satisfies

$$
|f(x)| \lesssim(1+|x|)^{M} \frac{e^{-s|x|^{p}} e^{-\varrho|x|}}{\sqrt{B(x)}}, \quad\left|\mathscr{F}_{A}(f)(\lambda)\right| \lesssim e^{-t|\lambda|^{q}},
$$

where $s, t$ are positive constants, $M$ is a nonnegative integer, $1<p<\infty$, and $\frac{1}{p}+\frac{1}{q}=1$. Here $B(x)$ is as in (1.2).

1) If $(s p)^{\frac{1}{p}}(t q)^{\frac{1}{q}}>1$ then $f=0$ almost everywhere.

2) If $(s p)^{\frac{1}{p}}(t q)^{\frac{1}{q}}=1$ and $p \neq 2$ (hence $q \neq 2$ ) then $f=0$ almost everywhere.

3) If $(s p)^{\frac{1}{p}}(t q)^{\frac{1}{q}}=1$ and $p=q=2$, i.e. $s t=\frac{1}{4}$, then, for all $M$, we have:

(i) For $\varrho=0, f(x)$ is, up to a constant, the heat kernel $h(t, x)$.

(ii) For $\varrho>0, f=0$ almost everywhere.

When $p=q=2$, we shall call Morgan's type theorem as the Hardy type theorem (see Corollary 6.3). The case $s t<\frac{1}{4}$ is included in Corollary 6.3. One can think of Morgan's type theorem as an intermediate result between the Paley-Winener theorem for $\mathscr{F}_{A}$, corresponding to $p=1$, and Hardy's type uncertainty principle.

Next we state a generalization of Hardy's type theorem (or Morgan's type theorem with $p=q=2$ ), where the uniform conditions are replaced by integrability conditions. 
Theorem F (Cowling-Price type; see Theorem 6.5). Let $f \in L^{2}(\mathbb{R}, A(x) d x)$ and assume that for positive real numbers $s, t$, and nonnegative integers $N_{1}, N_{2}$,

$$
\begin{aligned}
& \int_{\mathbb{R}}\left(\frac{|f(x)| e^{s|x|^{2}} e^{\varrho|x|\left(1-\frac{1}{p}\right)} B(x)^{\frac{1}{2}\left(1-\frac{1}{p}\right)}}{(1+|x|)^{N_{1}}}\right)^{p} A(x) d x<\infty, \\
& \int_{\mathbb{R}}\left(\frac{\left|\mathscr{F}_{A}(f)(\lambda)\right| e^{t|\lambda|^{2}}}{(1+|\lambda|)^{N_{2}}}\right)^{q} \xi(\lambda) d \lambda<\infty,
\end{aligned}
$$

where $1 \leq p, q \leq \infty$, and $B(x)$ is as in (1.2).

1) If $s t<\frac{1}{4}$, then there exist infinitely many linearly independent functions satisfying the above two conditions.

2) If $s t>\frac{1}{4}$ then $f(x)=0$ almost everywhere.

3) If $s t=\frac{1}{4}$ then

$$
f(x)=\sum_{k=0}^{n} c_{k} \Lambda_{A, x}^{(k)} h(t, x) \quad n<N_{2}-\frac{(2 \alpha+2)}{q} .
$$

In particular, if $\{(2 \alpha+2) / q\}<N_{2} \leq\{(2 \alpha+2) / q\}+1$ then $f(x)=c_{0} h(t, x)$, with:

(i) For $\varrho=0, c_{0}$ is an arbitrary constant whenever $N_{1}>\{(2 \alpha+2) / p\}$, otherwise $c_{0}=0$.

(ii) For $\varrho>0, c_{0}=0$.

For $p=q=\infty$, the Cowling-Price type theorem implies Hardy's type uncertainty principle.

\section{BACKGROUND}

The purpose of this section is to recall some results from the literature on the so-called Chébli transform. For more details we refer the reader to [9, 10, 11, 35, 36].

Throughout this paper we will denote by $A$ a function on $\mathbb{R}$ satisfying the following hypotheses:

(H1) $A(x)=|x|^{2 \alpha+1} B(x)$, where $\alpha>-\frac{1}{2}$ and $B$ is an even, positive and smooth function on $\mathbb{R}$ with $B(0)=1$.

(H2) $A$ is increasing and unbounded on $\mathbb{R}_{+}$.

(H3) $A^{\prime} / A$ is a decreasing and smooth function on $\mathbb{R}_{+}^{*}$, and hence the limit $2 \varrho:=$ $\lim _{x \rightarrow+\infty} A^{\prime}(x) / A(x) \geq 0$ exists.

Such a function $A$ is called a Chébli function. From (H1) it follows that

$$
\frac{A^{\prime}(x)}{A(x)}=\frac{2 \alpha+1}{x}+C(x), \quad \text { for } x \neq 0,
$$

where $C:=B^{\prime} / B$ is an odd and smooth function on $\mathbb{R}$. 
2.1. The Chébli transform. Let $\Delta_{A}$, or simply $\Delta$, be the following second order differential operator

$$
\Delta=\frac{d^{2}}{d x^{2}}+\frac{A^{\prime}(x)}{A(x)} \frac{d}{d x}
$$

For $\mu \in \mathbb{C}$, we consider the Cauchy problem

$$
\left\{\begin{array}{l}
\Delta f(x)=-\left(\mu^{2}+\varrho^{2}\right) f(x) \\
f(0)=1, \quad f^{\prime}(0)=0 .
\end{array}\right.
$$

In [11] the author proved that the system (2.3) has a unique solution $\varphi_{\mu}$. Moreover, $\varphi_{\mu}$ is an even smooth function on $\mathbb{R}$, and the map $\mu \mapsto \varphi_{\mu}(x)$ is analytic. We refer to [10, 36] for more details on $\varphi_{\mu}$.

For $f \in L^{1}\left(\mathbb{R}_{+}, A(x) d x\right)$, the Chébli transform of $f$ is given by

$$
\mathscr{F}_{\Delta}(f)(\mu):=\int_{\mathbb{R}_{+}} f(x) \varphi_{\mu}(x) A(x) d x, \quad|\operatorname{Im} \mu| \leq \varrho .
$$

The following Plancherel formula for $\mathscr{F}_{\Delta}$ was proved in [11].

Theorem 2.1. There exists a unique positive measure $\sigma$ with support $\mathbb{R}_{+}$such that $\mathscr{F}_{\Delta}$ induces an isometric isomorphism from $L^{2}\left(\mathbb{R}_{+}, A(x) d x\right)$ onto $L^{2}\left(\mathbb{R}_{+}, \sigma(d \mu)\right)$, and for any $f \in L^{1}\left(\mathbb{R}_{+}, A(x) d x\right) \cap L^{2}\left(\mathbb{R}_{+}, A(x) d x\right)$ we have

$$
\int_{\mathbb{R}_{+}}|f(x)|^{2} A(x) d x=\int_{\mathbb{R}_{+}}\left|\mathscr{F}_{\Delta}(f)(\mu)\right|^{2} \sigma(d \mu) .
$$

To have a nice behavior for the Plancherel measure $\sigma$ we need a further (growth) restriction on the function $A$. Following [35], we will assume that $A^{\prime} / A$ satisfies the additional hypothesis:

(H4) There exists a constant $\delta>0$ such that for all $x \in\left[x_{0}, \infty\right)$ (for some $x_{0}>0$ ),

$$
\frac{A^{\prime}(x)}{A(x)}= \begin{cases}2 \varrho+e^{-\delta x} D(x) & \text { if } \varrho>0, \\ \frac{2 \alpha+1}{x}+e^{-\delta x} D(x) & \text { if } \varrho=0,\end{cases}
$$

where $D$ being a smooth function bounded together with its derivatives.

In these circumstances, the Plancherel measure $\sigma$ is absolutely continuous with respect to the Lebesgue measure and has density $|c(\mu)|^{-2}$, where $c$ is a continuous function on $\mathbb{R}_{+}$and zero free on $\mathbb{R}_{+}^{*}$. Moreover, by [36, Proposition 6.1.12 and Corollary 6.1.5] (see also [6]), for $\mu \in \mathbb{C}$ we have:

(i) If $\varrho \geq 0$ and $\alpha>-1 / 2$, then $|c(\mu)|^{-2} \sim|\mu|^{2 \alpha+1}$ whenever $|\mu| \gg 1$.

(ii) If $\varrho>0$ and $\alpha>-1 / 2$, then $|c(\mu)|^{-2} \sim|\mu|^{2}$ whenever $|\mu| \ll 1$.

(iii) If $\varrho=0$ and $\alpha>0$, then $|c(\mu)|^{-2} \sim|\mu|^{2 \alpha+1}$ whenever $|\mu| \ll 1$.

In the literature, the function $c$ is called Harish-Chandra's function of the Laplacian $\Delta$. We refer to [7] for more details on the $c$-function.

Henceforth we will assume that Chébli's function $A$ satisfies the additional hypothesis (H4). In particular, it follows that for $|x|$ large enough: 
(i) $A(x) \sim e^{2 \varrho|x|}$ for $\varrho>0$.

(ii) $A(x) \sim|x|^{2 \alpha+1}$ for $\varrho=0$.

2.2. A family of differential-reflection operators. Consider the following differentialreflection operator

$$
\Lambda_{A} f(x)=f^{\prime}(x)+\frac{A^{\prime}(x)}{A(x)}\left(\frac{f(x)-f(-x)}{2}\right) .
$$

In view of the hypothesis $(\mathrm{H} 4)$ on $A^{\prime} / A$, the space $\mathscr{D}(\mathbb{R})$ (of smooth functions with compact support on $\mathbb{R}$ ) and the space $\mathscr{S}(\mathbb{R})$ (of Schwartz functions on $\mathbb{R}$ ) are invariant under the action of $\Lambda_{A}$.

For completeness' sake, the operator $\mathscr{L}_{A}:=\Lambda_{A}^{2}$ is given by

$$
\mathscr{L}_{A} f(x)=f^{\prime \prime}(x)+\left(\frac{A^{\prime}(x)}{A(x)}\right) f^{\prime}(x)+\left(\frac{A^{\prime}(x)}{A(x)}\right)^{\prime}\left(\frac{f(x)-f(-x)}{2}\right) .
$$

Let $\lambda \in \mathbb{C}$ and consider the differential-reflection equation

$$
\Lambda_{A} f(x)=i \lambda f(x)
$$

where $f: \mathbb{R} \rightarrow \mathbb{C}$.

Theorem 2.2. (see [4, Theorem 3.2]) The solution space of (2.8) is 1-dimensional for all $\lambda \in \mathbb{C}$. This solution space contains a (unique) function $\Psi(\lambda, \cdot)$ such that $\Psi(\lambda, 0)=1$. Further, for every $x \in \mathbb{R}$, the function $\lambda \mapsto \Psi(\lambda, x)$ is analytic on $\mathbb{C}$. More explicitly, we have:

$$
\Psi(\lambda, x)= \begin{cases}\varphi_{\mu}(x)+\frac{1}{i \lambda} \partial_{x} \varphi_{\mu}(x) & \text { if } \lambda \in \mathbb{C} \backslash\{0\}, \\ 1 & \text { if } \lambda=0,\end{cases}
$$

where $\mu$ satisfies the relation $\mu^{2}=\lambda^{2}-\varrho^{2}$. Furthermore, for all $\lambda, x \in \mathbb{R}$, we have

$$
|\Psi(\lambda, x)| \leq 1
$$

Example 2.3. Assume that $A(x)=A_{\alpha, \beta}(x)=(\sinh |x|)^{2 \alpha+1}(\cosh x)^{2 \beta+1}$ with $\alpha \geq \beta \geq$ $-1 / 2$ and $\alpha \neq-1 / 2$. In this example, the differential-reflection operator (2.6) coincides with the Heckman operator in one dimension,

$$
\Lambda_{A_{\alpha, \beta}} f(x)=f^{\prime}(x)+((2 \alpha+1) \operatorname{coth} x+(2 \beta+1) \tanh x)\left(\frac{f(x)-f(-x)}{2}\right) .
$$

The eigenfunction $\Psi(\lambda, x)$ is given by

$$
\begin{aligned}
\Psi(\lambda, x) & =\varphi_{\mu}^{(\alpha, \beta)}(x)+\frac{1}{i \lambda} \partial_{x} \varphi_{\mu}^{(\alpha, \beta)}(x) \\
& =\varphi_{\mu}^{(\alpha, \beta)}(x)+\frac{i \lambda}{4(\alpha+1)} \sinh (2 x) \varphi_{\mu}^{(\alpha+1, \beta+1)}(x)
\end{aligned}
$$

where $\varphi_{\mu}^{(\alpha, \beta)}$ denotes the Jacobi function, and $\mu^{2}=\lambda^{2}-(\alpha+\beta+1)^{2}$. 
2.3. An intertwining operator. In [34, Theorem 2.2] the author proved that there exists a unique automorphism $V_{A}$, or simply $V$, of $C^{\infty}(\mathbb{R})$ such that

$$
\Lambda_{A} \circ V=V \circ \frac{d}{d x} \text { and } V f(0)=f(0) .
$$

In particular, by the uniqueness of the solution $\Psi(\lambda, x)$, it follows that

$$
\Psi(\lambda, x)=V\left(e^{i \lambda \cdot}\right)(x) .
$$

Moreover, by [34, Theorem 3.2], there exists a positive kernel $\mathbb{K}$ so that

$$
V f(x)=\int_{|y|<|x|} \mathbb{K}(x, y) f(y) d y, \quad x \neq 0 .
$$

Henceforth, we will denote by ${ }^{\mathrm{t}} V$ the dual operator of $V$ in the sense that

$$
\int_{\mathbb{R}} V f(x) g(x) A(x) d x=\int_{\mathbb{R}} f(y){ }^{\mathrm{t}} V g(y) d y,
$$

for suitable $f$ and $g$. That is,

$$
{ }^{\mathrm{t}} V g(y)=\int_{|x|>|y|} \mathbb{K}(x, y) g(x) A(x) d x
$$

2.4. A generalized Fourier transform. On the space $L^{1}(\mathbb{R}, A(x) d x)$ we consider the generalized Fourier transform $\mathscr{F}_{A}$ defined by

$$
\mathscr{F}_{A}(f)(\lambda)=\int_{\mathbb{R}} f(x) \Psi(\lambda,-x) A(x) d x, \quad \lambda \in \mathbb{R} .
$$

In view of (2.11) and (2.13) we have

$$
\mathscr{F}_{A}=\mathscr{F}_{\text {euc }} \circ{ }^{\mathrm{t}} V \text {. }
$$

where $\mathscr{F}_{\text {euc }}$ is the Euclidean Fourier transform.

To state the alleged inverse transform of $\mathscr{F}_{A}$, let us introduce the following Plancherel measure

$$
\xi(\lambda) d \lambda:=\frac{|\lambda|}{\sqrt{\lambda^{2}-\varrho^{2}}\left|c\left(\sqrt{\lambda^{2}-\varrho^{2}}\right)\right|^{2}} \mathbf{1}_{\mathbb{R} \backslash]-\varrho, \varrho}(\lambda) d \lambda .
$$

Theorem 2.4. (see [4, Theorem 8.1 and 8.2])

1) If $f \in L^{1}(\mathbb{R}, A(x) d x)$ and $\mathscr{F}_{A}(f) \in L^{1}(\mathbb{R}, \xi(\lambda) d \lambda)$ then

$$
f(x)=\frac{1}{4} \int_{\mathbb{R}} \mathscr{F}_{A}(f)(\lambda) \Psi(\lambda, x) \xi(\lambda) d \lambda \quad \text { almost everywhere. }
$$

2) If $f \in L^{1}(\mathbb{R}, A(x) d x) \cap L^{2}(\mathbb{R}, A(x) d x)$, then $\mathscr{F}_{A}(f) \in L^{2}(\mathbb{R}, \xi(\lambda) d \lambda)$ and furthermore

$$
\int_{\mathbb{R}}|f(x)|^{2} A(x) d x=\frac{1}{4} \int_{\mathbb{R}}\left|\mathscr{F}_{A}(f)(\lambda)\right|^{2} \xi(\lambda) d \lambda .
$$

3) There exists a unique unitary operator on $L^{2}(\mathbb{R}, \xi(\lambda) d \lambda)$ that coincides with $(1 / 2) \mathscr{F}_{A}$ on $L^{1}(\mathbb{R}, A(x) d x) \cap L^{2}(\mathbb{R}, A(x) d x)$. 
For $0<p \leq 1$, denote by $\mathscr{S}_{p}(\mathbb{R})$ the space consisting of all functions $f \in C^{\infty}(\mathbb{R})$ such that

$$
\sigma_{s, k}^{(p)}(f):=\sup _{x \in \mathbb{R}}(|x|+1)^{s} \varphi_{0}(x)^{-2 / p}\left|f^{(k)}(x)\right|<\infty,
$$

for any $s \in \mathbb{N}$ and any $k \in \mathbb{N}$. The topology of $\mathscr{S}_{p}(\mathbb{R})$ is defined by the seminorms $\sigma_{s, k}^{(p)}$. We pin down that $\mathscr{S}_{p}(\mathbb{R})$ is a dense subspace of $L^{q}(\mathbb{R}, A(x) d x)$ for $p \leq q<\infty$, while it is not contained in $L^{q}(\mathbb{R}, A(x) d x)$ for $0<q<p$.

We set

$$
\mathbb{C}_{p}:=\left\{\lambda \in \mathbb{C}|| \operatorname{Im} \lambda \mid \leq \varrho\left(\frac{2}{p}-2\right)\right\} .
$$

Let $\mathscr{S}\left(\mathbb{C}_{p}\right)$ be the Schwartz space consists of all complex valued functions $h$ that are analytic in the interior of $\mathbb{C}_{p}$, and such that $h$ together with all its derivatives extend continuously to $\mathbb{C}_{p}$ and satisfy

$$
\tau_{t, \ell}^{(p)}(h):=\sup _{\lambda \in \mathbb{C}_{p}}(|\lambda|+1)^{t}\left|h^{(\ell)}(\lambda)\right|<\infty,
$$

for any $t \in \mathbb{N}$ and any $\ell \in \mathbb{N}$. The topology of $\mathscr{S}\left(\mathbb{C}_{p}\right)$ is defined by the seminorms $\tau_{t, \ell}^{(p)}$.

Using Anker's approach [1], we proved in [4, Theorem 9.6] the following result:

Theorem 2.5. For all $0<p \leq 1$, the Fourier transform $\mathscr{F}_{A}$ is a topological isomorphism between $\mathscr{S}_{p}(\mathbb{R})$ and $\mathscr{S}\left(\mathbb{C}_{p}\right)$.

2.5. A generalized convolution product. For fixed $x, y \in \mathbb{R}^{*}$ and for a suitable function $f$, we consider the following generalized translation operator (see [26, (5.3) and (6.1)] or [37, (4.4)])

$$
\tau_{y} f(x)=\int_{\mathbb{R}} f(z) d \mu_{x, y}(z),
$$

where

$$
\operatorname{supp}\left(\mu_{x, y}\right) \subset[-|x|-|y|,-|| x|-| y||] \cup[|| x|-| y||,|x|+|y|] .
$$

For $x=0$ or $y=0, \mu_{x, y}$ is nothing other than the $\delta$-measure. It is worth mentioning that

$$
\tau_{y} \Psi(\lambda, \cdot)(x)=\Psi(\lambda, x) \Psi(\lambda, y)
$$

For more details on the generalized translation operator $\tau_{y}$ we refer the reader to [37]. We recall the following result from [37, Theorem 4]. To simplify the notation, we will write $\|\cdot\|_{L_{x}^{p}}$ instead of $\|\cdot\|_{L^{p}(\mathbb{R}, A(x) d x)}$ where the lower-index in $L_{x}^{p}$ denotes the relevant variable.

Lemma 2.6. Let $f \in L^{p}(\mathbb{R}, A(x) d x)$ with $1 \leq p \leq \infty$. Then $\tau_{y} f(x)$ exists and is finite for almost every $x \in \mathbb{R}$. Moreover

$$
\tau_{y} f \in L^{p}(\mathbb{R}, A(x) d x) \quad \text { and } \quad\left\|\tau_{y} f\right\|_{L_{x}^{p}} \leq 2^{\left|1-\frac{2}{p}\right|}\|f\|_{L_{x}^{p}}
$$

The integral

$$
f *_{A} g(x):=\int_{\mathbb{R}} \tau_{-y} f(x) g(y) A(y) d y
$$

is called the generalized convolution product of $f$ and $g$. For the existence of $*_{A}$, one can impose different conditions on the functions $f$ and $g$. In particular, by standard 
arguments, one can prove the statement below. In order to be self-contained, we shall give a proof:

Proposition 2.7. 1) Assume that $1 \leq p, q, r \leq \infty$ satisfy $\frac{1}{p}+\frac{1}{q}-1=\frac{1}{r}$. Then, for every $f \in L^{p}(\mathbb{R}, A(x) d x)$ and $g \in L^{q}(\mathbb{R}, A(x) d x)$, we have $f *_{A} g \in L^{r}(\mathbb{R}, A(x) d x)$ and

$$
\left\|f *_{A} g\right\|_{L_{x}^{r}} \leq 2^{\left|1-\frac{2}{p}\right|}\|f\|_{L_{x}^{p}}\|g\|_{L_{x}^{q}} .
$$

2) Let $f \in L^{1}(\mathbb{R}, A(x) d x)$ and $g \in L^{1}(\mathbb{R}, A(x) d x)$. Then, $f *_{A} g \in L^{1}(\mathbb{R}, A(x) d x)$ and satisfies

$$
\mathscr{F}_{A}\left(f *_{A} g\right)(\lambda)=\mathscr{F}_{A}(f)(\lambda) \mathscr{F}_{A}(g)(\lambda) .
$$

3) Let $f \in L^{2}(\mathbb{R}, A(x) d x)$ and $g \in L^{2}(\mathbb{R}, A(x) d x)$. Then, $f *_{A} g \in L^{2}(\mathbb{R}, A(x) d x)$ if and only if $\mathscr{F}_{A}(f) \mathscr{F}_{A}(g) \in L^{2}(\mathbb{R}, \xi(\lambda) d \lambda)$ and

$$
\mathscr{F}_{A}\left(f *_{A} g\right)(\lambda)=\mathscr{F}_{A}(f)(\lambda) \mathscr{F}_{A}(g)(\lambda) .
$$

Proof. 1) If $r=\infty$ then $(1 / p)+(1 / q)=1$. Hence, by Hölder's inequality and Lemma 2.6 , $f *_{A} g(x)$ exists for each $x$ and $\left\|f *_{A} g\right\|_{L_{x}^{\infty}} \leq 2^{\left|1-\frac{2}{p}\right|}\|f\|_{L_{x}^{p}}\|g\|_{L_{x}^{q}}$. Next, suppose $1 \leq r<\infty$. Note that $p \leq r$ and $q \leq r$. Let $s=p(1-1 / q)=1-p / r$ and note that $0 \leq s<1$. Let $t=r / q$ and note that $1 \leq t<\infty$. Define $q^{\prime}$ by $(1 / q)+\left(1 / q^{\prime}\right)=1$ and note that $1<q^{\prime} \leq \infty$. Let

$$
h(x):=\int_{\mathbb{R}}\left|\tau_{-y} f(x) g(y)\right| A(y) d y=\int_{\mathbb{R}}\left|\tau_{x} f(-y)\right|^{1-s}|g(y)|\left|\tau_{x} f(-y)\right|^{s} A(y) d y .
$$

By Hölder's inequality we have

$$
h(x) \leq\left(\int_{\mathbb{R}}\left|\tau_{x} f(-y)\right|^{(1-s) q}|g(y)|^{q} A(y) d y\right)^{1 / q}\left\|\left|\tau_{x} f\right|^{s}\right\|_{L_{y}^{q^{\prime}}} .
$$

If $s=0$ then $q=1$. If $s \neq 0$ then $s q^{\prime}=p$. In either cases taking the $q^{\text {th }}$ power we obtain

$$
\begin{aligned}
h(x)^{q} & \leq\left(\int_{\mathbb{R}}\left|\tau_{x} f(-y)\right|^{(1-s) q}|g(y)|^{q} A(y) d y\right)\left\|\tau_{x} f\right\|_{L_{y}^{p}}^{s q} \\
& \leq 2^{s q\left|1-\frac{2}{p}\right|}\|f\|_{L_{y}^{p}}^{s q}\left(\int_{\mathbb{R}}\left|\tau_{x} f(-y)\right|^{(1-s) q}|g(y)|^{q} A(y) d y\right) .
\end{aligned}
$$

Thus, by the generalized Minkowski inequality we have

$$
\begin{aligned}
\|h\|_{L_{x}^{q t}}^{q} & =\left\|h^{q}\right\|_{L_{x}^{t}} \\
& \leq 2^{s q\left|1-\frac{2}{p}\right|}\|f\|_{L_{y}^{p}}^{s q}\left(\int_{\mathbb{R}}\left(\int_{\mathbb{R}}\left|\tau_{x} f(-y)\right|^{(1-s) q}|g(y)|^{q} A(y) d y\right)^{t} A(x) d x\right)^{1 / t} \\
& \leq 2^{s q\left|1-\frac{2}{p}\right|}\|f\|_{L_{y}^{p}}^{s q} \int_{\mathbb{R}}\left(\int_{\mathbb{R}}\left|\tau_{x} f(-y)\right|^{(1-s) q t}|g(y)|^{q t} A(x) d x\right)^{1 / t} A(y) d y \\
& \leq 2^{q\left|1-\frac{2}{p}\right|}\|f\|_{L_{y}^{p}}^{q}\|g\|_{L_{y}^{q}}^{q},
\end{aligned}
$$

since $q t=r$ and $(1-s) r=p$. Taking the $q^{\text {th }}$ root we obtain the statement.

2) For $f$ and $g$ in $L^{1}(\mathbb{R}, A(x) d x)$, it follows from the first part that $f *_{A} g \in L^{1}(\mathbb{R}, A(x) d x)$. In view of (2.22) the rest of the statement is a routine checking. 
3) The third statement can be proved by mimicking the argument used in [36, page 189] for the Chébli transform $\mathscr{F}_{\Delta}$. The details are left to the reader.

Henceforth, the notation $X \lesssim Y$ will be used to indicate that $X \leq C Y$ with a positive constant $C$ independent of significant quantities.

\section{A HeISENBERG TYPE INEQUALITY FOR $\mathscr{F}_{A}$}

Recall from (2.7) that $-\mathscr{L}_{A}$ is a positive self-adjoint operator on $L^{2}(\mathbb{R}, A(x) d x)$. Then, for $t>0, e^{t \mathscr{L}_{A}}$ defines an utltracontractive semigroup, called the heat semigroup, so that for all $f \in C_{c}^{\infty}(\mathbb{R}), u(x, t):=e^{t \mathscr{L}_{A}} f(x)$ solves the homogeneous heat equation

$$
\partial_{t} u(x, t)=\mathscr{L}_{A} u(x, t), \quad u(x, 0)=f(x) .
$$

The solution $e^{t \mathscr{L}_{A}} f(x)$ can be written as $e^{t \mathscr{L}_{A}} f(x)=h_{t} *_{A} f(x)$, where $h_{t}(x)=h(t, x):=$ $\mathscr{F}_{A}^{-1}\left(e^{-t \lambda^{2}}\right)(x)$ is the heat kernel.

We claim that $h_{t}$ belongs to the Schwartz space $\mathscr{S}_{p}(\mathbb{R})$ with $0<p \leq 1$ (see (2.19)), and therefore $h_{t} \in L^{q}(\mathbb{R}, A(x) d x)$ for all $0<q<\infty$. Indeed, as the function $\lambda \mapsto e^{-t \lambda^{2}}$ belongs to the Schwartz space $\mathscr{S}\left(\mathbb{C}_{p}\right)$, it follows from Theorem 2.5 that $h_{t} \in \mathscr{S}_{p}(\mathbb{R})$. The second part of the claim is due to the fact that $\mathscr{S}_{p}(\mathbb{R})$ is a dense subspace of $L^{q}(\mathbb{R}, A(x) d x)$ for $p \leq q<\infty$.

In view of the claim above, we may rewrite the heat kernel as

$$
h_{t}(x)=\frac{1}{4} \int_{\mathbb{R}} e^{-t \lambda^{2}} \Psi(\lambda, x) \xi(\lambda) d \lambda, \quad(t, x) \in \mathbb{R}_{+}^{*} \times \mathbb{R},
$$

where the Plancherel measure $\xi(\lambda) d \lambda$ is as in (2.16). By the expression (2.9) of $\Psi(\lambda, x)$, the integral in (3.1) becomes

$$
h_{t}(x)=\frac{1}{2} \int_{0}^{\infty} e^{-t\left(\mu^{2}+\varrho^{2}\right)} \varphi_{\mu}(x)|c(\mu)|^{-2} d \mu .
$$

The following statement is nothing other than Lemma 3.2 in [24].

Lemma 3.1. The following estimates hold:

1) For $\varrho>0$, we have

$$
\left\|h_{t}\right\|_{L_{x}^{2}} \sim \begin{cases}t^{-\frac{\alpha+1}{2}} & \text { for } 0<t \leq 1, \\ e^{-t \varrho^{2}} t^{-\frac{3}{4}} & \text { for } t>1 .\end{cases}
$$

2) For $\varrho=0$, and with the additional condition $\alpha>0$, we have

$$
\left\|h_{t}\right\|_{L_{x}^{2}} \sim t^{-\frac{\alpha+1}{2}}, \quad \text { for } t>0 .
$$

The next lemma is a cornerstone of the main result of this section. Define

$$
\mathbb{D}_{\varrho}:= \begin{cases}\{1\} & \text { if } \varrho=0, \\ \left(\frac{1}{2}, 1\right] & \text { if } 0<\varrho<1, \\ {\left[\frac{1}{2}, 1\right]} & \text { otherwise. }\end{cases}
$$


Lemma 3.2. Let $\gamma \in \mathbb{D}_{\varrho}$ and let $s>0$ so that $\gamma s<\alpha+1$ for $\varrho \geq 0$, and with the additional condition $\alpha>0$ for $\varrho=0$. Then, for all $f \in L^{2}(\mathbb{R}, A(x) d x)$, we have

$$
\left\|f *_{A} h_{t}\right\|_{L_{x}^{2}} \lesssim t^{-\frac{s}{2}}\left\||x|^{\gamma s} f\right\|_{L_{x}^{2}}
$$

Proof. Let us start with the case $\varrho>0$. For $r>0$, let $\chi_{r}$ be the characteristic function of the interval $[-r, r]$, and let $\chi_{r}^{\prime}=1-\chi_{r}$. Define the functions $f_{r}$ and $f^{r}$ by

$$
f_{r}:=f \chi_{r}, \quad f^{r}:=f \chi_{r}^{\prime} .
$$

Since $f^{r} \in L^{2}(\mathbb{R}, A(x) d x)$ and $h_{t} \in L^{1} \cap L^{2}(\mathbb{R}, A(x) d x)$, by Proposition 2.7.1 and 2.7.3, it follows that $h_{t} *_{A} f^{r} \in L^{2}(\mathbb{R}, A(x) d x)$ and $\mathscr{F}_{A}\left(h_{t} *_{A} f^{r}\right)=\mathscr{F}_{A}\left(h_{t}\right) \mathscr{F}_{A}\left(f^{r}\right)$. By using the Plancherel formula twice we get

$$
\begin{aligned}
\left\|h_{t} *_{A} f^{r}\right\|_{L_{x}^{2}} & =\left\|e^{-t \lambda^{2}} \mathscr{F}_{A}\left(f^{r}\right)\right\|_{L_{\lambda}^{2}} \\
& \leq\left\|\mathscr{F}_{A}\left(f^{r}\right)\right\|_{L_{\lambda}^{2}} \\
& \leq r^{-\gamma s}\left\||x|^{\gamma s} f\right\|_{L_{x}^{2}}
\end{aligned}
$$

since $\gamma s>0$. Above, the notation $\|\cdot\|_{L_{\lambda}^{2}}$ stands for $\|\cdot\|_{L^{2}(\mathbb{R}, \xi(\lambda) d \lambda)}$.

On the other hand, by appealing to Proposition 2.7.1 we have

$$
\begin{aligned}
\left\|h_{t} *_{A} f_{r}\right\|_{L_{x}^{2}} & \leq\left\|h_{t}\right\|_{L_{x}^{2}}\left\|f_{r}\right\|_{L_{x}^{1}} \\
& \leq\left\|h_{t}\right\|_{L_{x}^{2}}\left\||x|^{\gamma s} f\right\|_{L_{x}^{2}}\left(2 \int_{0}^{r} x^{-2 \gamma s} A(x) d x\right)^{\frac{1}{2}} .
\end{aligned}
$$

Further, one may check that under the assumption on $\gamma s$, we have

$$
\left(\int_{0}^{r} x^{-2 \gamma s} A(x) d x\right)^{\frac{1}{2}} \lesssim r^{-\gamma s} V(r),
$$

where

$$
V(r)= \begin{cases}r^{\alpha+1} & \text { for } r \leq 1, \\ e^{\varrho r} \sqrt{r} & \text { for } r>1 .\end{cases}
$$

This is due to the fact that, for $\varrho>0, A(x) \sim e^{2 \varrho|x|}$ when $|x| \gg 1$ and $A(x) \sim|x|^{2 \alpha+1}$ when $|x| \ll 1$.

Consequently,

$$
\begin{aligned}
\left\|h_{t} *_{A} f\right\|_{L_{x}^{2}} & \leq\left\|h_{t} *_{A} f^{r}\right\|_{L_{x}^{2}}+\left\|h_{t} *_{A} f_{r}\right\|_{L_{x}^{2}} \\
& \lesssim r^{-\gamma s}\left\||x|^{\gamma s} f\right\|_{L_{x}^{2}}\left(1+V(r)\left\|h_{t}\right\|_{L_{x}^{2}}\right) .
\end{aligned}
$$

Now, choosing $r=t^{\frac{1}{2 \gamma}}$ we obtain

$$
\left\|h_{t} *_{A} f\right\|_{L_{x}^{2}} \lesssim t^{-\frac{s}{2}}\left\||x|^{\gamma s} f\right\|_{L_{x}^{2}}\left(1+V\left(t^{\frac{1}{2 \gamma}}\right)\left\|h_{t}\right\|_{L_{x}^{2}}\right) .
$$

Using the estimate (3.2) for $\left\|h_{t}\right\|_{L_{x}^{2}}$ we deduce that

$$
V\left(t^{\frac{1}{2 \gamma}}\right)\left\|h_{t}\right\|_{L_{x}^{2}} \sim \begin{cases}t^{\frac{\alpha+1}{2}\left(\frac{1}{\gamma}-1\right)} & \text { for } t \leq 1, \\ t^{\frac{1}{4}\left(\frac{1}{\gamma}-3\right)} e^{-\varrho t\left(\varrho-t^{(1-2 \gamma) /(2 \gamma)}\right)} & \text { for } t>1 .\end{cases}
$$

Thus, $V\left(t^{\frac{1}{2 \gamma}}\right)\left\|h_{t}\right\|_{L_{x}^{2}}$ is bounded for all $\gamma \in \mathbb{D}_{\varrho}$ and for all $t>0$. 
The case $\varrho=0$ runs similarly.

The principal result of this section is the following:

Theorem 3.3. (Heisenberg's inequality) Let $r, s$ be positive constants and let $\gamma \in \mathbb{D}_{\varrho}$. Then, for all $f \in L^{2}(\mathbb{R}, A(x) d x)$ we have

$$
\left\||x|^{\gamma s} f\right\|_{L_{x}^{2}}^{\frac{r}{r+s}}\left\||\lambda|^{r} \mathscr{F}_{A}(f)\right\|_{L_{\lambda}^{2}}^{\frac{s}{r+s}} \gtrsim\|f\|_{L_{x}^{2}}
$$

Proof. We will give the proof for $\varrho>0$; the case $\varrho=0$ runs similarly. Let us assume first that $\gamma \in \mathbb{D}_{\varrho}$ and $s>0$ so that $\gamma s<\alpha+1$. By Lemma 3.2 and by the Plancherel formula (2.18), we have

$$
\begin{aligned}
\|f\|_{L_{x}^{2}} & \leq\left\|h_{t} *_{A} f\right\|_{L_{x}^{2}}+\left\|f-h_{t} *_{A} f\right\|_{L_{x}^{2}} \\
& \lesssim t^{-\frac{s}{2}}\left\||x|^{\gamma s} f\right\|_{L_{x}^{2}}+\left\|\left(1-e^{-t \lambda^{2}}\right)\left(t|\lambda|^{2}\right)^{-\frac{r}{2}}\left(t|\lambda|^{2}\right)^{\frac{r}{2}} \mathscr{F}_{A}(f)\right\|_{L_{\lambda}^{2}}
\end{aligned}
$$

Suppose that $r \leq 2$. Then the function $u \mapsto\left(1-e^{-u}\right) u^{-\frac{r}{2}}$ is bounded for all $u \geq 0$, and therefore

$$
\|f\|_{L_{x}^{2}} \lesssim t^{-\frac{s}{2}}\left\||x|^{\gamma s} f\right\|_{L_{x}^{2}}+t^{\frac{r}{2}}\left\||\lambda|^{r} \mathscr{F}_{A}(f)\right\|_{L_{\lambda}^{2}} .
$$

The minimum value of the right hand-side of (3.8) (as a function of $t>0$ ) is, up to a constant,

$$
\left\||x|^{\gamma s} f\right\|_{L_{x}^{2}}^{\frac{r}{r+s}}\left\||\lambda|^{r} \mathscr{F}_{A}(f)\right\|_{L_{\lambda}^{2}}^{\frac{s}{r+s}}
$$

This finishes the proof in the case where $\gamma s<\alpha+1$ and $r \leq 2$.

Suppose that $r>2$. Consider a real number $r^{\prime}$ so that $0<r^{\prime} \leq 2<r$. Obviously $u^{r^{\prime}} \leq 1+u^{r}$ for all $u \geq 0$. In particular, for $u=|\lambda| / \sqrt{\varepsilon}$ with $\varepsilon>0$, we have

$$
\left(\frac{|\lambda|^{2}}{\varepsilon}\right)^{\frac{r^{\prime}}{2}} \leq 1+\left(\frac{|\lambda|^{2}}{\varepsilon}\right)^{\frac{r}{2}}
$$

It follows that

$$
\begin{aligned}
\left\||\lambda|^{r^{\prime}} \mathscr{F}_{A}(f)\right\|_{L_{\lambda}^{2}} & \leq \varepsilon^{\frac{r^{\prime}}{2}}\left\|\mathscr{F}_{A}(f)\right\|_{L_{\lambda}^{2}}+\varepsilon^{\frac{r^{\prime}-r}{2}}\left\||\lambda|^{r} \mathscr{F}_{A}(f)\right\|_{L_{\lambda}^{2}} \\
& =\varepsilon^{\frac{r^{\prime}}{2}}\|f\|_{L_{x}^{2}}+\varepsilon^{\frac{r^{\prime}-r}{2}}\left\||\lambda|^{r} \mathscr{F}_{A}(f)\right\|_{L_{\lambda}^{2}} .
\end{aligned}
$$

Optimizing (3.9) in $\varepsilon$ we get

$$
\left\||\lambda|^{r^{\prime}} \mathscr{F}_{A}(f)\right\|_{L_{\lambda}^{2}} \lesssim\|f\|_{L_{x}^{2}}^{1-\frac{r^{\prime}}{r}}\left\||\lambda|^{r} \mathscr{F}_{A}(f)\right\|_{L_{\lambda}^{2}}^{\frac{r^{\prime}}{r}}
$$

Since $r^{\prime} \leq 2$, the inequality (3.10) implies

$$
\begin{aligned}
\|f\|_{L_{x}^{2}} & \lesssim\left\||x|^{\gamma s} f\right\|_{L_{x}^{2}}^{\frac{r^{\prime}}{r^{\prime}+s}}\left\||\lambda|^{r^{\prime}} \mathscr{F}_{A}(f)\right\|_{L_{\lambda}^{2}}^{\frac{s}{r^{\prime}+s}} \\
& \lesssim\left\||x|^{\gamma s} f\right\|_{L_{x}^{2}}^{\frac{r^{\prime}}{r^{\prime}+s}}\|f\|_{L_{x}^{2}}^{\left(1-\frac{r^{\prime}}{r}\right) \frac{s}{r^{\prime}+s}}\left\||\lambda|^{r} \mathscr{F}_{A}(f)\right\|_{L_{\lambda}^{2}}^{\left(\frac{r^{\prime}}{r}\right)\left(\frac{s}{r^{\prime}+s}\right)} .
\end{aligned}
$$

That is

$$
\|f\|_{L_{x}^{2}} \lesssim\left\||x|^{\gamma s} f\right\|_{L_{x}^{2}}^{\frac{r}{r+s}}\left\||\lambda|^{r} \mathscr{F}_{A}(f)\right\|_{L_{\lambda}^{2}}^{\frac{s}{r+s}}
$$

This finishes the proof when $\gamma s<\alpha+1$ and $r>2$. 
Next we discuss the remaining case $\gamma s \geq \alpha+1$. Let $s^{\prime}>0$ such that $\gamma s^{\prime}<\alpha+1 \leq \gamma s$. Since $\gamma s^{\prime}<\gamma s$ it follows that

$$
\left(\frac{|x|}{\varepsilon}\right)^{\gamma s^{\prime}} \leq 1+\left(\frac{|x|}{\varepsilon}\right)^{\gamma s}, \quad \forall \varepsilon>0 .
$$

Now the statement can be proven in a fashion similar to what was used in the case $\gamma s<\alpha+1$ and $r>2$.

\section{A local UNCERTAinty INEQUALITy FOR $\mathscr{F}_{A}$}

In this section we will establish a local uncertainty inequality for the Fourier transform $\mathscr{F}_{A}$. We will prove that if $f$ is highly localized, then $\mathscr{F}_{A}(f)$ cannot be concentrated in a small neighborhood of two or more widely separated points.

The following lemma is needed for later use.

Lemma 4.1. Assume that $\lambda \in \mathbb{R}$. For all $x \in \mathbb{R},|\Psi(\lambda, x)|=1$ if and only if $\lambda=0$.

Proof. From (2.9) we have $\Psi(0, x)=1$ for all $x \in \mathbb{R}$. Now let us prove the opposite direction. Since $\Psi(\lambda, \cdot)$ is a solution to $(2.8)$, it follows that

$$
\partial_{x} \Psi(\lambda, x)=-\frac{A^{\prime}(x)}{2 A(x)}(\Psi(\lambda, x)-\Psi(\lambda,-x))+i \lambda \Psi(\lambda, x) .
$$

Using the oddness of the function $A^{\prime}$, we deduce that

$$
\partial_{x}\{\Psi(\lambda,-x)\}=-\frac{A^{\prime}(x)}{2 A(x)}(\Psi(\lambda,-x)-\Psi(\lambda, x))-i \lambda \Psi(\lambda,-x),
$$

and by consequence

$$
\partial_{x}\{\overline{\Psi(\lambda,-x)}\}=-\frac{A^{\prime}(x)}{2 A(x)}(\overline{\Psi(\lambda,-x)}-\overline{\Psi(\lambda, x)})+i \lambda \overline{\Psi(\lambda,-x)} .
$$

Therefore

$$
\begin{aligned}
\partial_{x}|\Psi(\lambda,-x)|^{2}=- & \frac{A^{\prime}(x)}{2 A(x)} \\
& \{(\Psi(\lambda,-x)-\Psi(\lambda, x)) \overline{\Psi(\lambda,-x)}+(\overline{\Psi(\lambda,-x)}-\overline{\Psi(\lambda, x)}) \Psi(\lambda,-x)\} .
\end{aligned}
$$

Similarly we have

$$
\begin{aligned}
\partial_{x}|\Psi(\lambda, x)|^{2}=-\frac{A^{\prime}(x)}{2 A(x)} & \\
& \{(\Psi(\lambda, x)-\Psi(\lambda,-x)) \overline{\Psi(\lambda, x)}+(\overline{\Psi(\lambda, x)}-\overline{\Psi(\lambda,-x)}) \Psi(\lambda, x)\} .
\end{aligned}
$$

Hence, for every $x \in \mathbb{R}$, we get

$$
\partial_{x}\left\{|\Psi(\lambda, x)|^{2}+|\Psi(\lambda,-x)|^{2}\right\}=-\frac{A^{\prime}(x)}{A(x)}|\Psi(\lambda,-x)-\Psi(\lambda, x)|^{2} .
$$

Assume that there exists a $\lambda_{0} \in \mathbb{R}$ such that $\left|\Psi\left(\lambda_{0}, x\right)\right|=1$ for all $x \in \mathbb{R}$. From (4.1) it follows that $\left|\Psi\left(\lambda_{0},-x\right)-\Psi\left(\lambda_{0}, x\right)\right|=0$. That is $\Psi\left(\lambda_{0}, x\right)=\Psi\left(\lambda_{0},-x\right)$ for every $x \in \mathbb{R}$. 
By the expression (2.9) of $\Psi(\lambda, x)$ we deduce that $\varphi_{\mu_{0}}^{\prime}(x)=0$ for all $x \in \mathbb{R}$, where $\mu_{0}^{2}=$ $\lambda_{0}^{2}-\varrho^{2}$. In view of the definition (2.2) of the Laplacian $\Delta$, saying $\varphi_{\mu_{0}}^{\prime}(x)=0$ is equivalent to $\Delta \varphi_{\mu_{0}}(x)=0$. Thus, knowing that $\Delta \varphi_{\mu_{0}}(x)=-\lambda_{0}^{2} \varphi_{\mu_{0}}(x)$, we have $\lambda_{0}^{2} \varphi_{\mu_{0}}(x)=0$ for all $x \in \mathbb{R}$. Since $\varphi_{\mu_{0}}(0)=1$, we deduce that $\lambda_{0}=0$.

We now come to the main result of this section.

Theorem 4.2. (Local uncertainty inequality) Let $s$ be a positive constant so that $s<$ $\alpha+1$, and let $E \subseteq \mathbb{R} \backslash]-\varrho, \varrho[$ be a measurable set such that $0<\xi(E)<\infty$. Then, for all nonzero function $f \in L^{2}(\mathbb{R}, A(x) d x)$, we have

$$
\left\|f *_{A} w_{E}\right\|_{L_{x}^{2}} \supsetneqq c_{s, E}\left\|w_{E}\right\|_{L_{x}^{2}}^{\frac{s}{\alpha+1}}\left\||x|^{s} f\right\|_{L_{x}^{2}},
$$

where $w_{E}:=\mathscr{F}_{A}^{-1} \chi_{E} \in L^{2}(\mathbb{R}, A(x) d x)$.

Proof. For $r>0$, let $\chi_{r}$ be the characteristic function of the interval $\left.I_{r}=\right]-r, r[$ and let $\chi_{r}^{\prime}=1-\chi_{r}$. By Minkowski's inequality and the Plancherel formula (2.18), we have

$$
\begin{aligned}
\left\|\mathscr{F}_{A}(f) \chi_{E}\right\|_{L_{\lambda}^{2}} & \leq\left\|\mathscr{F}_{A}\left(f \chi_{r}\right) \chi_{E}\right\|_{L_{\lambda}^{2}}+\left\|\mathscr{F}_{A}\left(f \chi_{r}^{\prime}\right) \chi_{E}\right\|_{L_{\lambda}^{2}} \\
& \leq \sqrt{\xi(E)}\left\|\mathscr{F}_{A}\left(f \chi_{r}\right)\right\|_{L_{\lambda}^{\infty}}+\left\|\mathscr{F}_{A}\left(f \chi_{r}^{\prime}\right)\right\|_{L_{\lambda}^{2}} \\
& \leq \sqrt{\xi(E)}\left\|f \chi_{r}\right\|_{L_{x}^{1}}+\left\|f \chi_{r}^{\prime}\right\|_{L_{x}^{2}} .
\end{aligned}
$$

By Hölder's inequality, it is clear that

$$
\left\|f \chi_{r}\right\|_{L_{x}^{1}} \leq\left(2 \int_{0}^{r} t^{-2 s} A(t) d t\right)^{\frac{1}{2}}\left\||x|^{s} f\right\|_{L_{x}^{2}} .
$$

The above integral between parentheses converges, since $s<\alpha+1$. Further, we have

$$
\begin{aligned}
\left\|f \chi_{r}^{\prime}\right\|_{L_{x}^{2}} & \leq\left\||x|^{-s} \chi_{r}^{\prime}\right\|_{L_{x}^{\infty}}\left\||x|^{s} f\right\|_{L_{x}^{2}} \\
& \leq r^{-s}\left\||x|^{s} f\right\|_{L_{x}^{2}} .
\end{aligned}
$$

In view of (4.3) and (4.4), the inequality (4.2) becomes

$$
\left\|\mathscr{F}_{A}(f) \chi_{E}\right\|_{L_{\lambda}^{2}} \leq \Theta_{s, E}(r)\left\||x|^{s} f\right\|_{L_{x}^{2}}
$$

for every $r>0$, where

$$
\begin{aligned}
\Theta_{a, E}(r) & :=r^{-s}+\sqrt{\xi(E)}\left(2 \int_{0}^{r} t^{-2 s} A(t) d t\right)^{\frac{1}{2}} \\
& \leq r^{-s}\left(1+\sqrt{\xi(E)} \frac{r^{\alpha+1}}{\sqrt{1+\alpha-s}}\left(\sup _{t \in[0, r]} B(t)\right)^{\frac{1}{2}}\right) .
\end{aligned}
$$

Above we have used the fact that $A(x)=|x|^{2 \alpha+1} B(x)$, where $\alpha>-\frac{1}{2}$ and $B$ is an even, positive and smooth function on $\mathbb{R}$. Let us choose $r=r_{0}:=\xi(E)^{-\frac{1}{2 \alpha+2}}$. Thus, the inequality (4.5) reduces to

$$
\left\|\mathscr{F}_{A}(f) \chi_{E}\right\|_{L_{\lambda}^{2}} \leq\left(1+\frac{1}{\sqrt{1+\alpha-s}}\left(\sup _{t \in\left[0, r_{0}\right]} B(t)\right)^{\frac{1}{2}}\right) \xi(E)^{\frac{s}{2 \alpha+2}}\left\||x|^{s} f\right\|_{L_{x}^{2}} .
$$


Next we will prove that the inequality (4.6) is strict. To do so, let us assume that there exists a nonzero function $\tilde{f} \in L^{2}(\mathbb{R}, A(x) d x)$ for which

$$
\left\|\mathscr{F}_{A}(\tilde{f}) \chi_{E}\right\|_{L_{\lambda}^{2}}=\left(1+\frac{1}{\sqrt{1+\alpha-s}}\left(\sup _{t \in\left[0, r_{0}\right]} B(t)\right)^{\frac{1}{2}}\right) \xi(E)^{\frac{s}{2 \alpha+2}}\left\||x|^{S} \tilde{f}\right\|_{L_{x}^{2}} .
$$

Using (4.7), one can check the following identities:

$$
\begin{aligned}
& \left\|\mathscr{F}_{A}\left(\tilde{f} \chi_{r_{0}}\right) \chi_{E}\right\|_{L_{\lambda}^{2}}=\sqrt{\xi(E)}\left\|\mathscr{F}_{A}\left(\tilde{f} \chi_{r_{0}}\right)\right\|_{L_{\lambda}^{\infty}}, \\
& \left\|\tilde{f} \chi_{r_{0}}\right\|_{L_{x}^{1}}=\left\|\mathscr{F}_{A}\left(\tilde{f} \chi_{r_{0}}\right)\right\|_{L_{\lambda}^{\infty}}, \\
& \left\|\tilde{f} \chi_{r_{0}}\right\|_{L_{x}^{1}}=\left\||x|^{-s} \chi_{r_{0}}\right\|_{L_{x}^{2}}\left\||x|^{s} \tilde{f}\right\|_{L_{x}^{2} .}
\end{aligned}
$$

The strategy is to use the identities (4.9) and (4.10) to identify the function $\tilde{f}$, and then to employ the identity (4.8) to derive a contradiction.

First, by Cauchy-Schwarz, the identity (4.10) holds true if and only if

$$
|\tilde{f}(x)|=c|x|^{-2 s} \chi_{r_{0}}(x),
$$

for some constant $c>0$. That is, there exists a complex valued function $\phi$ on $\mathbb{R}$ satisfying $|\phi(x)|=1$ and such that

$$
\tilde{f}(x)=c \phi(x)|x|^{-2 s} \chi_{r_{0}}(x) .
$$

In view of (4.11), the identity (4.9) becomes

$$
\|\tilde{f}\|_{L_{x}^{1}}=\left\|\mathscr{F}_{A}(\tilde{f})\right\|_{L_{\lambda}^{\infty}} .
$$

Then, there exists $\lambda_{0} \in \mathbb{R}$ such that

$$
\|\tilde{f}\|_{L_{x}^{1}}=\left|\mathscr{F}_{A}(\tilde{f})\left(\lambda_{0}\right)\right|,
$$

which implies

$$
\|\tilde{f}\|_{L_{x}^{1}}=e^{-i \theta_{0}} \mathscr{F}_{A}(\tilde{f})\left(\lambda_{0}\right),
$$

for some $\theta_{0} \in \mathbb{R}$. By the expression (4.11) of $\tilde{f}$, the identity (4.13) is explicitly given by:

$$
c \int_{\mathbb{R}}|x|^{-2 s} \chi_{r_{0}}(x)\left(1-e^{-i \theta_{0}} \phi(x) \Psi\left(\lambda_{0},-x\right)\right) A(x) d x=0 .
$$

In particular we get

$$
\int_{\mathbb{R}}|x|^{-2 s} \chi_{r_{0}}(x)\left(1-\operatorname{Re}\left\{e^{-i \theta_{0}} \phi(x) \Psi\left(\lambda_{0},-x\right)\right\}\right) A(x) d x=0 .
$$

Since $\left|e^{-i \theta_{0}} \phi(x) \Psi\left(\lambda_{0},-x\right)\right| \leq 1$, which is essentially due to the fact that $\left|\Psi\left(\lambda_{0},-x\right)\right| \leq 1$, it follows that $\left|\operatorname{Re}\left\{e^{-i \theta_{0}} \phi(x) \Psi\left(\lambda_{0},-x\right)\right\}\right| \leq 1$. By this elementary observation, the identity (4.15) implies

$$
1-\operatorname{Re}\left\{e^{-i \theta_{0}} \phi(x) \Psi\left(\lambda_{0},-x\right)\right\}=0
$$

for almost every $x \in \mathbb{R}$. Using again the fact that $\left|e^{-i \theta_{0}} \phi(x) \Psi\left(\lambda_{0},-x\right)\right| \leq 1$, we conclude that

$$
e^{-i \theta_{0}} \phi(x) \Psi\left(\lambda_{0},-x\right)=1
$$

for almost every $x \in \mathbb{R}$. By a standard argument, the identity (4.16) holds true for all $x \in \mathbb{R}$. Since $|\phi(x)|=1$, we deduce from (4.16) that $\left|\Psi\left(\lambda_{0},-x\right)\right|=1$ for all $x \in \mathbb{R}$. In 
view of Lemma 4.1, this is true if and only if $\lambda_{0}=0$. Consequently, the identity (4.16) becomes

$$
\phi(x)=e^{i \theta_{0}}
$$

and therefore (see (4.11))

$$
\tilde{f}(x)=c e^{i \theta_{0}}|x|^{-2 s} \chi_{r_{0}}(x) .
$$

We claim that the function $\tilde{f}$ found above is not accommodate in the identity (4.8). Indeed, substituting (4.17) back in (4.8) we see that

$$
\left\|\mathscr{F}_{A}(\tilde{f}) \chi_{E}\right\|_{L_{\lambda}^{2}}^{2}=\xi(E)\left\|\mathscr{F}_{A}(\tilde{f})\right\|_{L_{\lambda}^{\infty}}^{2} .
$$

That is

$$
\int_{E}\left(\left\|\mathscr{F}_{A}(\tilde{f})\right\|_{L_{\lambda}^{\infty}}^{2}-\left|\mathscr{F}_{A}(\tilde{f})(\lambda)\right|^{2}\right) \xi(\lambda) d \lambda=0
$$

Since by assumption $\xi(E)>0$, it follows that the integrand in (4.18) vanishes for almost every $\lambda \in E$, i.e.

$$
\left|\mathscr{F}_{A}(\tilde{f})(\lambda)\right|=\left\|\mathscr{F}_{A}(\tilde{f})\right\|_{L_{\lambda}^{\infty}}
$$

for almost every $\lambda \in E$. Recall from above that $\left\|\mathscr{F}_{A}(\tilde{f})\right\|_{L_{\lambda}^{\infty}}=\left|\mathscr{F}_{A}(\tilde{f})(0)\right|$. Thus, (4.19) reads $\left|\mathscr{F}_{A}(\tilde{f})(\lambda)\right|=\left|\mathscr{F}_{A}(\tilde{f})(0)\right|$ for almost every $\lambda \in E$. Hence, there exists a measurable function $\kappa$ such that

$$
\mathscr{F}_{A}(\tilde{f})(\lambda)=\kappa(\lambda) \mathscr{F}_{A}(\tilde{f})(0)
$$

with $|\kappa(\lambda)|=1$. In view of the expression (4.17) of $\tilde{f}$, we may rewrite the above identity as

$$
c e^{i \theta_{0}} \int_{\mathbb{R}}|x|^{-2 s} \chi_{r_{0}}(x)(\kappa(\lambda)-\Psi(\lambda,-x)) A(x) d x=0 .
$$

In a similar fashion to what was used in the previous integral (4.14), we deduce that for almost every $\lambda \in E$ and for every $x \in \mathbb{R}$

$$
\kappa(\lambda)-\Psi(\lambda,-x)=0 .
$$

Since $|\kappa(\lambda)|=1$, we obtain $|\Psi(\lambda,-x)|=1$ for almost every $\lambda \in E$ (which is $\subset \mathbb{R} \backslash]-\varrho, \varrho[$ ).

(i) If $\varrho>0$, then, by Lemma 4.1 , this cannot be true. It follows that the identity (4.18) does not hold. That is our assumption (4.7) cannot operate.

(ii) If $\varrho=0$, then Lemma 4.1 reduces the set of $\lambda$ 's to $\{0\}$. That is $\xi(E)=0$, which contradicts the hypothesis on the set $E$. Thus, (4.18) does not remain true. In other words, our assumption (4.7) cannot last.

In conclusion, the inequality in (4.6) is strict.

\section{A Beurling type THEOREM}

In this section we establish the analogue of Beurling's theorem for the Fourier transform $\mathscr{F}_{A}$. In the limit case $\alpha=-1 / 2$, with $B(x)=1$ for all $x \in \mathbb{R}$, our theorem coincides with the Bonami-Demange-Jaming result in one dimension [5], which generalizes the original Beurling's theorem. 
Theorem 5.1. Let $f \in L^{2}(\mathbb{R}, A(x) d x)$ satisfy

$$
\int_{\mathbb{R}} \int_{\mathbb{R}} \frac{|f(x)|\left|\mathscr{F}_{A}(f)(\lambda)\right| e^{|x||\lambda|}}{(1+|x|+|\lambda|)^{N}} A(x) d x \xi(\lambda) d \lambda<\infty,
$$

for some nonnegative integer $N$. Then $f$ is of the form

$$
f(x)=\sum_{k=0}^{n} c_{k} \Lambda_{A, x}^{(k)} h(t, x) \quad n<\frac{N-(2 \alpha+2)}{2},
$$

where $c_{k}$ are complex constants, and the subscript in $\Lambda_{A, x}^{(k)} h(t, x)$ denotes the relevant variable in the $k$-th power of the operator $\Lambda_{A}$ applied to the heat kernel $h(t, x)$ for some $t>0$. In particular, if $N \leq 2 \alpha+2$ then $f=0$.

We will first prove the following statement.

Proposition 5.2. Under the same assumptions as in Theorem 5.1. we have:

1) The function $f$ belongs to the space $L^{1}(\mathbb{R}, A(x) d x)$.

2) The function ${ }^{t} V f$ belongs to the space $L^{p}(\mathbb{R})$ for $1 \leq p \leq \infty$.

Proof. 1) On one hand, since $f \in L^{2}(\mathbb{R}, A(x) d x)$ then $f$ is locally integrable on $\mathbb{R}$ with respect to the measure $A(x) d x$. On the other hand, from the master condition (5.1) it follows that

$$
\int_{\mathbb{R}} \frac{|f(x)| e^{|x||\lambda|}}{(1+|x|)^{N}} A(x) d x<\infty
$$

for almost every $\lambda \in \mathbb{R} \backslash]-\varrho, \varrho\left[\right.$ so that $\mathscr{F}_{A}(f)(\lambda) \neq 0$. We pin down that if $\mathscr{F}_{A}(f)(\lambda)=0$ for almost every $\lambda \in \mathbb{R} \backslash]-\varrho, \varrho[$, then by the Plancherel Theorem 2.4 .3 we get $f=0$ almost everywhere.

Let $\lambda_{0} \in \mathbb{R} \backslash[-\varrho, \varrho]$ such that $\mathscr{F}_{A}(f)\left(\lambda_{0}\right) \neq 0$, and rewrite the inequality $(5.3)$ for $\lambda=\lambda_{0}$. Since $e^{|x|\left|\lambda_{0}\right|} /(1+|x|)^{N} \gg 1$ for $|x|$ large enough, and since $f \in L_{\text {loc }}^{1}(\mathbb{R}, A(x) d x)$, we deduce that $f \in L^{1}(\mathbb{R}, A(x) d x)$.

2) By assumption and the first statement, we have $f \in L^{1} \cap L^{2}(\mathbb{R}, A(x) d x)$. We claim that

$$
{ }^{\mathrm{t}} V f \in L^{1}(\mathbb{R}) \cap L^{\infty}(\mathbb{R})
$$

Indeed,

$$
\begin{aligned}
\int_{\mathbb{R}}\left|{ }^{t} V f(y)\right| d y & \leq \int_{\mathbb{R}} \int_{|x|>|y|} \mathbb{K}(x, y)|f(x)| A(x) d x d y \\
& \leq \int_{\mathbb{R}}|f(x)|\left(\int_{|y|<|x|} \mathbb{K}(x, y) d y\right) A(x) d x \\
& =\|f\|_{L_{x}^{1}}<\infty .
\end{aligned}
$$

Above we have used the fact that $\mathbb{K}$ is positive and that

$$
\int_{|y|<|x|} \mathbb{K}(x, y) d y=\Psi(0, x)=1,
$$

(see (2.12) and (2.11)). Thus ${ }^{t} V f \in L^{1}(\mathbb{R})$. 
Next we will prove that ${ }^{\mathrm{t}} V f \in L^{\infty}(\mathbb{R})$. By the master condition (5.1) we deduce that there exists $x_{0} \in \mathbb{R}^{*}$ such that

$$
\int_{\mathbb{R}} \frac{\left|\mathscr{F}_{A}(f)(\lambda)\right| e^{\left|x_{0}\right||\lambda|}}{(1+|\lambda|)^{N}} \xi(\lambda) d \lambda<\infty .
$$

Since $e^{\left|x_{0}\right||\lambda|} /(1+|\lambda|)^{N} \gg 1$ for $|\lambda|$ large enough (say $\left.|\lambda|>R\right)$, it follows that

$$
\int_{|\lambda|>R}\left|\mathscr{F}_{A}(f)(\lambda)\right| \xi(\lambda) d \lambda<\infty .
$$

On the other hand, as $f \in L^{1} \cap L^{2}(\mathbb{R}, A(x) d x)$, by the Plancherel Theorem 2.4.2, we deduce that $\mathscr{F}_{A}(f) \in L^{2}(\mathbb{R}, \xi(\lambda) d \lambda)$. Hence $\mathscr{F}_{A}(f) \in L_{\text {loc }}^{1}(\mathbb{R}, \xi(\lambda) d \lambda)$. In conclusion, $\mathscr{F}_{A}(f) \in L^{1}(\mathbb{R}, \xi(\lambda) d \lambda)$. This conclusion leads to $\mathscr{F}_{A}(f) \in L^{1}(\mathbb{R})$. In fact, let us split

$$
\int_{\mathbb{R}}\left|\mathscr{F}_{A}(f)(\lambda)\right| d \lambda=I_{1}+I_{2}+I_{3}
$$

according to

$$
\int_{\mathbb{R}}=\int_{|\lambda|<\rho+1}+\int_{\rho+1 \leq|\lambda| \leq L}+\int_{|\lambda|>L} .
$$

Here $L>0$ is chosen large enough so that $\xi(\lambda)>1$ for $|\lambda|>L$.

Since $\mathscr{F}_{A}(f) \in L^{1}(\mathbb{R}, \xi(\lambda) d \lambda)$, we deduce that

$$
I_{3} \leq \int_{|\lambda|>L}\left|\mathscr{F}_{A}(f)(\lambda)\right| \xi(\lambda) d \lambda<\infty .
$$

For $I_{2}$, observe that the function $\lambda \mapsto \frac{|\lambda|}{\sqrt{\lambda^{2}-\rho^{2}}\left|c\left(\sqrt{\lambda^{2}-\rho^{2}}\right)\right|^{2}}$ is continuous and bounded for $\rho+1 \leq|\lambda| \leq L$. Thus, there exist $a, b>0$ such that $a \leq \xi(\lambda) \leq b$ for $\rho+1 \leq|\lambda| \leq L$, and therefore

$$
I_{2} \leq \frac{1}{a} \int_{\rho+1 \leq|\lambda| \leq L}\left|\mathscr{F}_{A}(f)(\lambda)\right| \xi(\lambda) d \lambda<\infty .
$$

Before studying the finiteness of the integral $I_{1}$, we point down that $\mathscr{F}_{A}(f) \in L^{\infty}(\mathbb{R})$. The latter fact is due to the identity $\mathscr{F}_{A}(f)=\mathscr{F}_{\text {euc }}{ }^{\mathrm{t}}{ }^{\mathrm{t}} V f$ (since $f \in L^{1} \cap L^{2}(\mathbb{R}, A(x) d x)$ ) and that ${ }^{\mathrm{t}} V f \in L^{1}(\mathbb{R})$. Hence

$$
I_{1} \leq 2(\rho+1)\left\|\mathscr{F}_{A}(f)\right\|_{L^{\infty}(\mathbb{R})}<\infty .
$$

In conclusion,

$$
\int_{\mathbb{R}}\left|\mathscr{F}_{A}(f)(\lambda)\right| d \lambda=I_{1}+I_{2}+I_{3}<\infty,
$$

i.e. $\mathscr{F}_{A}(f) \in L^{1}(\mathbb{R})$.

Since ${ }^{t} V f \in L^{1}(\mathbb{R})$ and $\mathscr{F}_{\text {euc }}{ }^{\mathrm{t}} V f=\mathscr{F}_{A}(f) \in L^{1}(\mathbb{R})$, by the inversion formula for Euclidean Fourier transform we deduce that ${ }^{\mathrm{t}} V f \in L^{\infty}(\mathbb{R})$. This finishes the proof of our claim (5.4).

In the light of (5.4), the second statement of Proposition 5.2 follows from Lyapunov inequality.

Now we turn our attention to Beurling's Theorem 5.1. 
Proof of Theorem 5.1. We will show that the master condition (5.1) implies

$$
\int_{\mathbb{R}} \int_{\mathbb{R}} \frac{{ }^{\mathrm{t}} V f(x)|| \mathscr{F}_{A}(f)(\lambda) \mid e^{|x||\lambda|}}{(1+|x|+|\lambda|)^{N}} d x \xi(\lambda) d \lambda<\infty .
$$

Choose a nonzero $\lambda_{0} \in \mathbb{R} \backslash[-\varrho, \varrho]$ such that $\mathscr{F}_{A}(f)\left(\lambda_{0}\right) \neq 0$, and write the integral (5.7) as the superposition of

$$
I:=\int_{\mathbb{R}} \int_{|\lambda| \leq\left|\lambda_{0}\right|} \frac{\left|{ }^{t} V f(x)\right|\left|\mathscr{F}_{A}(f)(\lambda)\right| e^{|x||\lambda|}}{(1+|x|+|\lambda|)^{N}} \xi(\lambda) d \lambda d x
$$

and

$$
J:=\int_{\mathbb{R}} \int_{|\lambda|>\left|\lambda_{0}\right|} \frac{\left|{ }^{\mathrm{t}} V f(x)\right|\left|\mathscr{F}_{A}(f)(\lambda)\right| e^{|x|}|\lambda|}{(1+|x|+|\lambda|)^{N}} \xi(\lambda) d \lambda d x
$$

Obviously we have

$$
I \lesssim \int_{\mathbb{R}} \frac{\left|{ }^{t} V f(x)\right| e^{|x|\left|\lambda_{0}\right|}}{(1+|x|)^{N}} d x
$$

We write the integral on the right hand-side of (5.8) as the superposition of $I_{1}$ and $I_{2}$ where

$$
I_{1}:=\int_{|x| \leq \frac{N}{\lambda_{0} \mid}} \frac{\left|{ }^{t} V f(x)\right| e^{|x|\left|\lambda_{0}\right|}}{(1+|x|)^{N}} d x, \quad I_{2}:=\int_{|x|>\frac{N}{\lambda_{0} \mid}} \frac{\left|{ }^{t} V f(x)\right| e^{|x|\left|\lambda_{0}\right|}}{(1+|x|)^{N}} d x .
$$

Recall from Proposition 5.2 that $\left\|{ }^{t} V f\right\|_{L^{1}(\mathbb{R})}<\infty$. Then

$$
I_{1} \lesssim \int_{|x| \leq \frac{N}{\lambda_{0} \mid}}\left|{ }^{\mathrm{t}} V f(x)\right| d x \leq\left\|\left.\right|^{\mathrm{t}} V f\right\|_{L^{1}(\mathbb{R})}<\infty .
$$

For $\ell>0$, the function $\varphi_{\ell}(s)=\frac{e^{\ell s}}{(1+s)^{N}}$ is monotonically increasing for $s>\frac{N}{\ell}$. Thus, by (5.3) one concludes

$$
\begin{aligned}
I_{2} & \leq \int_{|x|>\frac{N}{\lambda_{0} \mid}} \frac{e^{|x|\left|\lambda_{0}\right|}}{(1+|x|)^{N}}\left(\int_{|y|>|x|} \mathbb{K}(y, x)|f(y)| A(y) d y\right) d x \\
& \leq \int_{|y|>\frac{N}{\lambda_{0} \mid}} \frac{|f(y)| e^{|y|\left|\lambda_{0}\right|}}{(1+|y|)^{N}}\left(\int_{|x|<|y|} \mathbb{K}(y, x) d x\right) A(y) d y \\
& \leq \int_{\mathbb{R}} \frac{|f(y)| e^{|y|\left|\lambda_{0}\right|}}{(1+|y|)^{N}} A(y) d y<\infty
\end{aligned}
$$

To show that $J$ is finite, let us assume first that $\left|\lambda_{0}\right| \leq N$, and let us write the integral $J$ as $J=J_{1}+J_{2}+J_{3}$ where

$$
\begin{aligned}
& J_{1}:=\int_{|x| \leq \frac{N}{\left|\lambda_{0}\right|}} \int_{\left|\lambda_{0}\right|<|\lambda| \leq N} \frac{\left.\right|^{\mathrm{t} V} V(x)|| \mathscr{F}_{A}(f)(\lambda) \mid e^{|x||\lambda|}}{(1+|x|+|\lambda|)^{N}} \xi(\lambda) d \lambda d x, \\
& J_{2}:=\int_{|x|>\frac{N}{\left|\lambda_{0}\right|}} \int_{\left|\lambda_{0}\right|<|\lambda| \leq N} \frac{\left.\right|^{\mathrm{t}} V f(x)|| \mathscr{F}_{A}(f)(\lambda)\left|e^{|x|}\right| \lambda \mid}{(1+|x|+|\lambda|)^{N}} \xi(\lambda) d \lambda d x,
\end{aligned}
$$


and

$$
J_{3}:=\int_{\mathbb{R}} \int_{|\lambda|>N} \frac{\left|{ }^{t} V f(x)\right|\left|\mathscr{F}_{A}(f)(\lambda)\right| e^{|x||\lambda|}}{(1+|x|+|\lambda|)^{N}} \xi(\lambda) d \lambda d x .
$$

Clearly we have

$$
\left.J_{1} \lesssim \int_{|x| \leq \frac{N}{\Lambda_{0} \mid}}\right|^{\mathrm{t}} V f(x) \mid d x<\infty
$$

For $\ell>0$, the function

$$
\psi_{\ell}(s)=\frac{e^{\ell s}}{(1+s+\ell)^{N}}
$$

is monotonically increasing for $s>\frac{N}{\ell}$. Therefore, by the master condition (5.1) one concludes

$$
\begin{aligned}
J_{2} \leq & \int_{\left|\lambda_{0}\right|<|\lambda| \leq N}\left|\mathscr{F}_{A}(f)(\lambda)\right| \times \\
& \left(\int_{|x|>\frac{N}{\Lambda_{0} \mid}} \frac{e^{|x| \lambda \mid}}{(1+|x|+|\lambda|)^{N}}\left\{\int_{|y|>|x|} \mathbb{K}(y, x)|f(y)| A(y) d y\right\} d x\right) \xi(\lambda) d \lambda \\
\leq & \int_{\left|\lambda_{0}\right|<|\lambda| \leq N}\left|\mathscr{F}_{A}(f)(\lambda)\right|\left(\int_{|y|>\frac{N}{\mu_{0} \mid}} \frac{e^{|y||\lambda|}}{(1+|y|+|\lambda|)^{N}}|f(y)| A(y) d y\right) \xi(\lambda) d \lambda \\
\leq & \int_{\mathbb{R}} \int_{\mathbb{R}}|f(y)|\left|\mathscr{F}_{A}(f)(\lambda)\right| \frac{e^{|y||\lambda|}}{(1+|y|+|\lambda|)^{N}} A(y) d y \xi(\lambda) d \lambda<\infty .
\end{aligned}
$$

Let $\psi_{\ell}$ be as in (5.9). For $\ell>N$, the function $\psi_{\ell}$ is monotonically increasing for all $s \geq 0$. Hence, using again the master condition (5.1) to deduce that

$$
\begin{aligned}
J_{3} & \leq \int_{|\lambda|>N}\left|\mathscr{F}_{A}(f)(\lambda)\right|\left(\int_{\mathbb{R}} \frac{e^{|x||\lambda|}}{(1+|x|+|\lambda|)^{N}}\left\{\int_{|y|>|x|} \mathbb{K}(y, x)|f(y)| A(y) d y\right\} d x\right) \xi(\lambda) d \lambda \\
& \leq \int_{|\lambda|>N}\left|\mathscr{F}_{A}(f)(\lambda)\right|\left(\int_{\mathbb{R}} \frac{e^{|y| \lambda \mid}}{(1+|y|+|\lambda|)^{N}}|f(y)| A(y) d y\right) \xi(\lambda) d \lambda \\
& \leq \int_{|\lambda|>N} \int_{\mathbb{R}}\left|\mathscr{F}_{A}(f)(\lambda)\right||f(y)| \frac{e^{|y||\lambda|}}{(1+|y|+|\lambda|)^{N}} A(y) d y \xi(\lambda) d \lambda<\infty .
\end{aligned}
$$

In conclusion, the integral $J$ is finite when $\left|\lambda_{0}\right| \leq N$. Now we consider the case when $\left|\lambda_{0}\right|>N$. However, in this case

$$
J=\int_{\mathbb{R}} \int_{|\lambda|>\left|\lambda_{0}\right|>N} \frac{\left.\right|^{t} V f(x)|| \mathscr{F}_{A}(f)(\lambda) \mid e^{|x||\lambda|}}{(1+|x|+|\lambda|)^{N}} \xi(\lambda) d \lambda d x \leq J_{3}<\infty .
$$

This finishes the proof of the claim (5.7).

Next we will prove that in $(5.7)$ the measure $\xi(\lambda) d \lambda$ can be replaced by $d \lambda$, i.e.

$$
\int_{\mathbb{R}} \int_{\mathbb{R}} \frac{\left|{ }^{\mathrm{t}} V f(x)\right|\left|\mathscr{F}_{A}(f)(\lambda)\right| e^{|x||\lambda|}}{(1+|x|+|\lambda|)^{N}} d \lambda d x<\infty
$$


By the asymptotic behavior of $|c(\lambda)|^{-2}$ near infinity (see Subsection 2.1 ), we can choose a suitable large $R>0$ such that $\xi(\lambda)>1$ for $|\lambda|>R$. Hence we have

$$
\begin{aligned}
& \int_{\mathbb{R}} \int_{\mathbb{R}} \frac{{ }^{t} V f(x)|| \mathscr{F}_{A}(f)(\lambda) \mid e^{|x||\lambda|}}{(1+|x|+|\lambda|)^{N}} d \lambda d x \\
\leq & \int_{\mathbb{R}} \int_{|\lambda| \leq R} \frac{\left|{ }^{t} V f(x)\right|\left|\mathscr{F}_{A}(f)(\lambda)\right| e^{|x|}|\lambda|}{(1+|x|+|\lambda|)^{N}} d \lambda d x+\int_{\mathbb{R}} \int_{|\lambda|>R} \frac{\left|{ }^{t} V f(x)\right|\left|\mathscr{F}_{A}(f)(\lambda)\right| e^{|x||\lambda|}}{(1+|x|+|\lambda|)^{N}} \xi(\lambda) d \lambda d x .
\end{aligned}
$$

The inequality (5.7) implies that the second integral on the right hand-side is finite. To prove the finiteness of the first integral on the right hand-side, one can follow the same approach we used above for the finiteness of the integral $I$ (see (5.8)).

The final step in the proof is to use the fact that $\mathscr{F}_{A}=\mathscr{F}_{\text {euc }}{ }^{\mathrm{t}}{ }^{\mathrm{t}} \mathrm{V}$, see (2.15). In order to use the Euclidean version of Beurling's theorem, recall from Proposition 5.2 that ${ }^{\mathrm{t}} V(f) \in L^{2}(\mathbb{R})$.

Now, in view of (5.10), the Euclidean version of Beurling's theorem implies

$$
{ }^{\mathrm{t}} V f(x)=P(x) e^{-\frac{|x|^{2}}{4 r}}, \quad \forall x \in \mathbb{R},
$$

where $r>0$ and $P$ is a polynomial of degree $n<\frac{N-1}{2}$ (see [5, Theorem 1.1]). In particular, if $N \leq 1$ then $f=0$ almost everywhere. Using the well known fact that the Euclidean Fourier transform of $e^{-\frac{t^{2}}{2 a^{2}}}$ is given by $a e^{-\frac{\xi^{2} a^{2}}{2}}$, we deduce from (5.11) that

$$
\mathscr{F}_{A}(f)(\lambda)=\mathscr{F}_{\text {euc }} \circ{ }^{\mathrm{t}} V(f)(\lambda)=Q(\lambda) e^{-r|\lambda|^{2}},
$$

where $Q$ is a polynomial of the same degree of $P$.

Substituting (5.11) and (5.12) back in (5.7) we see that the integrand in (5.7) is

$$
\frac{|P(x)||Q(\lambda)|}{(1+|x|+|\lambda|)^{N}} e^{-\left(\frac{|x|}{2 \sqrt{r}}-\sqrt{r} \mid \lambda\right)^{2}} \xi(\lambda) \text {. }
$$

Using the asymptotic behavior of $|c(\lambda)|^{-2}$ for $|\lambda|$ large (see Subsection 2.1 ), it is easy to check that $\operatorname{deg}(P)(=\operatorname{deg}(Q))<\frac{N-(2 \alpha+2)}{2}$ as otherwise the integrand in $(5.13)$ is not integrable in the neighborhood of the lines $|x|=2 r|\lambda|$. Hence, the condition $n<\frac{N-1}{2}$ should be replaced by $n<\frac{N-(2 \alpha+2)}{2}$ (which is $<\frac{N-1}{2}$ ). In particular, if $N \leq 2 \alpha+2$, then $f=0$ almost everywhere.

For $N>2 \alpha+2$, the injectivity of the Fourier transform $\mathscr{F}_{A}$ together with (5.12) imply that $f$ is of the form

$$
f(x)=\sum_{k=0}^{n} c_{k} \Lambda_{A, x}^{(k)} h(r, x) \quad n<\frac{N-(2 \alpha+2)}{2},
$$

where $c_{k}$ are complex constants, and the subscript in $\Lambda_{A, x}^{(k)} h(r, x)$ denotes the relevant variable in the $k$-th power of the operator $\Lambda_{A}$ applied to the heat kernel $h(r, x)$ for some $r>0$. 


\section{Applications of Beurling's type theorem}

In this section we will show that Beurling's type Theorem 5.1 has several interesting consequences. More precisely, we will prove how theorems of Gelfand-Shilov, Morgan's, Hardy's, and Cowling-Price type all follow from Theorem 5.1.

Theorem 6.1 (Gelfand-Shilov type). Let $f \in L^{2}(\mathbb{R}, A(x) d x)$ and $N$ be a nonnegative integer. Assume that

$$
\begin{aligned}
& \int_{\mathbb{R}} \frac{|f(x)| e^{\frac{(2 s)}{p}|x|^{p}}}{(1+|x|)^{N}} A(x) d x<\infty, \\
& \int_{\mathbb{R}} \frac{\left|\mathscr{F}_{A}(f)(\lambda)\right| e^{\frac{(2 t)^{q}}{q}|\lambda|^{q}}}{(1+|\lambda|)^{N}} \xi(\lambda) d \lambda<\infty,
\end{aligned}
$$

where $1<p<\infty, \frac{1}{p}+\frac{1}{q}=1$, and $s$, are positive constants.

1) If st $>\frac{1}{4}$ then $f=0$ almost everywhere.

2) If $s t=\frac{1}{4}$ and $p \neq 2$ (hence $q \neq 2$ ) then $f=0$ almost everywhere.

3) If $s t=\frac{1}{4}$ and $p=q=2$, then

$$
f(x)=\sum_{k=0}^{n} c_{k} \Lambda_{A, x}^{(k)} h\left(2 t^{2}, x\right) \quad n<N-(2 \alpha+2),
$$

where $c_{k}$ are complex constants, and the subscript in $\Lambda_{A, x}^{(k)} h\left(2 t^{2}, x\right)$ denotes the relevant variable in the $k$-th power of the operator $\Lambda_{A}$ applied to the heat kernel $h\left(2 t^{2}, x\right)$. In particular,

(i) If $N \leq 2 \alpha+2$ then $f=0$ almost everywhere.

(ii) If $2 \alpha+2<N \leq 2 \alpha+3$ then $f(x)=c_{0} h\left(2 t^{2}, x\right)$, where $c_{0}$ is an arbitrary constant for $\varrho=0$, and $c_{0}=0$ for $\varrho>0$.

Proof. Since $4 s t|x||\lambda| \leq \frac{(2 s)^{p}}{p}|x|^{p}+\frac{(2 t)^{q}}{q}|\lambda|^{q}$, the conditions (GS1) and (GS2) imply

$$
\begin{aligned}
& \int_{\mathbb{R}} \int_{\mathbb{R}} \frac{|f(x)|\left|\mathscr{F}_{A}(f)(\lambda)\right|}{(1+|x|+|\lambda|)^{2 N}} e^{4 s t|x||\lambda|} A(x) d x \xi(\lambda) d \lambda \\
& \leq \int_{\mathbb{R}} \int_{\mathbb{R}} \frac{|f(x)| e^{\frac{(2 s) p}{p}|x|^{p}}}{(1+|x|)^{N}} \frac{\left|\mathscr{F}_{A}(f)(\lambda)\right| e^{\frac{(2 t)^{q}}{q}|\lambda|^{q}}}{(1+|\lambda|)^{N}} A(x) d x \xi(\lambda) d \lambda<\infty .
\end{aligned}
$$

As $4 s t \geq 1$, it follows that $f$ satisfies Beurling's master condition (5.1) with $2 N$ instead of $N$. Thus, $f \in L^{1}(\mathbb{R}, A(x) d x)$ and we have

$$
\begin{gathered}
{ }^{\mathrm{t}} V f(x)=P(x) e^{-\frac{|x|^{2}}{4 a},} \\
\mathscr{F}_{A}(f)(\lambda)=Q(\lambda) e^{-a|\lambda|^{2},}
\end{gathered}
$$

where $a>0$, and $P$ and $Q$ are two polynomials of the same degree $n<N-(\alpha+1)$. Further, by same arguments as in the proof of Theorem 5.1, the inequality (6.2) implies 
that $f$ satisfies

$$
\int_{\mathbb{R}} \int_{\mathbb{R}} \frac{\left|{ }^{t} V f(x)\right|\left|\mathscr{F}_{A}(f)(\lambda)\right| e^{4 s t|x|}|\lambda|}{(1+|x|+|\lambda|)^{2 N}} d x d \lambda<\infty .
$$

Furthermore, we claim that

$$
\int_{\mathbb{R}} \frac{\left|{ }^{t} V f(x)\right| e^{\frac{(2 s)^{p}}{p}|x|^{p}}}{(1+|x|)^{N}} d x<\infty
$$

Indeed,

$$
\int_{\mathbb{R}} \frac{\left|{ }^{\mathrm{t}} V f(x)\right| e^{\frac{(2 . s)^{p}}{p}|x|^{p}}}{(1+|x|)^{N}} d x \leq \int_{\mathbb{R}} \frac{e^{\frac{\left(2 . s p^{p}\right.}{p}|x|^{p}}}{(1+|x|)^{N}}\left(\int_{|y|>|x|} \mathbb{K}(y, x)|f(y)| A(y) d y\right) d x
$$

Choose a suitable large $R>0$ for which the function $u \mapsto \frac{e^{\frac{(2 s) p}{p} u^{p}}}{(1+u)^{N}}$ is increasing for $u>R$. Thus

$$
\begin{aligned}
\int_{\mathbb{R}} \frac{\left|{ }^{t} V f(x)\right| e^{\frac{(2 s)}{p}|x|^{p}}}{(1+|x|)^{N}} d x \leq & \int_{|x| \leq R} \frac{e^{\frac{(2 s) p^{p}}{p}|x|^{p}}}{(1+|x|)^{N}}\left(\int_{|y|>|x|} \mathbb{K}(y, x)|f(y)| A(y) d y\right) d x \\
& +\int_{|y|>R} \frac{|f(y)| e^{\frac{(2 s) p}{p}|y|^{p}}}{(1+|y|)^{N}}\left(\int_{|y|>|x|} \mathbb{K}(y, x) d x\right) A(y) d y .
\end{aligned}
$$

By the condition (GS1), the second integral on the right hand-side is finite, while the first one is bounded (up to a constant) by

$$
\begin{aligned}
& \int_{|x| \leq R}\left(\int_{|y|>|x|} \mathbb{K}(y, x)|f(y)| A(y) d y\right) d x \\
& \text { which is } \leq \int_{\mathbb{R}}|f(y)| A(y) d y<\infty,
\end{aligned}
$$

since $f \in L^{1}(\mathbb{R}, A(x) d x)$. This finishes the proof of the claim (6.6).

Substituting (6.3) and (6.4) in (6.5) we see that the integrand is

$$
\frac{|P(x)||Q(\lambda)|}{(1+|x|+|\lambda|)^{2 N}} e^{-\left(\frac{|x|}{2 \sqrt{a}}-\sqrt{a}|\lambda|\right)^{2}} e^{(4 s t-1)|x||\lambda|} .
$$

Hence, if $s t>\frac{1}{4}$ then the integrand grows exponentially in the neighborhood of the lines $|x|=2 a|\lambda|$ and the integral in (6.5) diverges. Now we assume that $s t=\frac{1}{4}$. By replacing $(6.3)$ and (6.4) in (6.6) and (GS2) respectively, we obtain

$$
\begin{gathered}
\int_{\mathbb{R}} \frac{|P(x)| e^{-\frac{|x|^{2}}{4 a}} e^{\frac{(2 s)^{p}}{p}|x|^{p}}}{(1+|x|)^{N}} d x<\infty, \\
\int_{\mathbb{R}} \frac{|Q(\lambda)| e^{-a|\lambda|^{2}} e^{\frac{(2 t)^{q}}{q}|\lambda|^{q}}}{(1+|\lambda|)^{N}} \xi(\lambda) d \lambda<\infty .
\end{gathered}
$$

However, when $p>2$ (and hence $q<2$ ), respectively $q>2$ (and hence $p<2$ ), the relation (6.7), respectively (6.8), does not hold true unless $P=0$, respectively $Q=0$. Thus, we are left with the case $p=q=2$. Recall that we are assuming here $s t=\frac{1}{4}$. 
In these circumstances, the finiteness of the integrals (6.7) and (6.8) imply that $a=2 t^{2}$. Moreover, the inequality (6.8) implies that the degree $n$ of $Q$ should satisfy $n<N-$ $(2 \alpha+2)$ (which is $<N-(\alpha+1)$ ). Now the injectivity of the Fourier transform $\mathscr{F}_{A}$ and the fact that $\mathscr{F}_{A}(f)(\lambda)=Q(\lambda) e^{-a|\lambda|^{2}}($ see $(\underline{6.4}))$, gives

$$
f(x)=\sum_{k=0}^{n} c_{k} \Lambda_{A, x}^{(k)} h\left(2 t^{2}, x\right) \quad n<N-(2 \alpha+2),
$$

where $c_{k}$ are complex constants and the subscript in $\Lambda_{A, x}^{(k)} h\left(2 t^{2}, x\right)$ denotes the relevant variable in the $k$-th power of the operator $\Lambda_{A}$ applied to the heat kernel $h\left(2 t^{2}, x\right)$.

Assume that $2 \alpha+2<N \leq 2 \alpha+3$. Then $f(x)=c_{0} h\left(2 t^{2}, x\right)$. At this stage of the proof we will distinguish the two cases $\varrho>0$ and $\varrho=0$. Recall that $A(x)=|x|^{2 \alpha+1} B(x)$, where $\alpha>-\frac{1}{2}$ and $B$ is an even, positive and smooth function on $\mathbb{R}$ with $B(0)=1$. From [16, Theorem 3.1] there exist two real numbers $\mu_{1}$ and $\mu_{2}$ such that for $t>0$ and $x \in \mathbb{R}$,

$$
\frac{e^{\mu_{1} t}}{2^{\alpha} \Gamma(\alpha+1)(2 t)^{\alpha+1}} \frac{e^{-\frac{|x|^{2}}{4 t}}}{\sqrt{B(x)}} \leq h(t, x) \leq \frac{e^{\mu_{2} t}}{2^{\alpha} \Gamma(\alpha+1)(2 t)^{\alpha+1}} \frac{e^{-\frac{|x|^{2}}{4 t}}}{\sqrt{B(x)}} .
$$

By making use of the fact that for $|x|$ large, $A(x) \sim e^{2 \varrho|x|}$ for $\varrho>0$ and $A(x) \sim|x|^{2 \alpha+1}$ for $\varrho=0$, we deduce from $(\underline{6.10})$ that $c_{0}=0$ for $\varrho>0$, otherwise the heat kernel fails to accommodate the inequality (GS1). For $\varrho=0$, the estimates $(6.10)$ show that $h\left(2 t^{2}, x\right)$ satisfies the condition (GS1) whenever $N>2 \alpha+2$ and no matter what value of $c_{0}$ is used.

The following uncertainty principle follows partially from the above theorem.

Theorem 6.2 (Morgan's type). Suppose a measurable function $f: \mathbb{R} \rightarrow \mathbb{C}$ satisfies

$$
\begin{array}{ll}
|f(x)| \lesssim(1+|x|)^{M} \frac{e^{-a|x|^{p}} e^{-\varrho|x|}}{\sqrt{B(x)}} & \forall x \in \mathbb{R}, \\
\left|\mathscr{F}_{A}(f)(\lambda)\right| \lesssim e^{-b|\lambda|^{q}} & \forall \lambda \in \mathbb{C},
\end{array}
$$

where $a, b$ are positive constants, $M$ is a nonnegative integer, $1<p<\infty$, and $\frac{1}{p}+\frac{1}{q}=1$.

1) If $(a p)^{\frac{1}{p}}(b q)^{\frac{1}{q}}>1$ then $f=0$ almost everywhere.

2) If $(a p)^{\frac{1}{p}}(b q)^{\frac{1}{q}}=1$ and $p \neq 2$ (hence $q \neq 2$ ) then $f=0$ almost everywhere.

3) If $(a p)^{\frac{1}{p}}(b q)^{\frac{1}{q}}=1$ and $p=q=2$, i.e. ab $=\frac{1}{4}$, then, for all $M \geq 0$, we have:

(i) For $\varrho=0, f(x)$ is, up to a constant, the heat kernel $h(b, x)$.

(ii) For $\varrho>0, f=0$ almost everywhere.

Proof. From the condition (M1) it follows that $f \in L^{2}(\mathbb{R}, A(x) d x)$. For $a=\frac{(2 s)^{p}}{p}$ and $b=\frac{(2 t)^{q}}{q}$, the function $f$ satisfies the condition (GS1) and (GS2) in Theorem 6.1 for suitable $N>0$. The condition $(a p)^{\frac{1}{p}}(b q)^{\frac{1}{q}} \geq 1$ becomes $4 s t \geq 1$. Hence, the statements 1) and 2) are direct consequences of Theorem 6.1. For the statement 3), by mimicking the proof of Theorem 6.1 one concludes that

$$
\mathscr{F}_{A}(f)(\lambda)=R(\lambda) e^{-2 t^{2}|\lambda|^{2}}=R(\lambda) e^{-b|\lambda|^{2}} .
$$


Making use of the condition (M2) we should have $R(\lambda)=$ const. Hence, up to a constant, $\mathscr{F}_{A}(f)(\lambda)$ coincides with $e^{-b|\lambda|^{2}}$, which is nothing other than the Fourier transform of the heat kernel $h(b, \cdot)$. That is

$$
f(x)=c_{0} h(b, x)
$$

for some constant $c_{0} \in \mathbb{C}$. Finally, from $(\underline{6.10})$ we deduce that, no matter what value of $M$ in the condition ( $\overline{\mathrm{M} 1}$ ) is used, for $\varrho>0$, the constant $c_{0}=0$ since the heat kernel does not obey the condition (M1), while for $\varrho=0$, the heat kernel accommodates the inequality (M1).

When $p=q=2$, Morgan's type theorem is nothing other than the following Hardy type theorem.

Corollary 6.3 (Hardy's type). Suppose a measurable function $f: \mathbb{R} \rightarrow \mathbb{C}$ satisfies

$$
\begin{aligned}
& |f(x)| \lesssim(1+|x|)^{M} \frac{e^{-a|x|^{2}} e^{-\varrho|x|}}{\sqrt{B(x)}} \quad \forall x \in \mathbb{R}, \\
& \left|\mathscr{F}_{A}(f)(\lambda)\right| \lesssim e^{-b|\lambda|^{2}} \quad \forall \lambda \in \mathbb{C},
\end{aligned}
$$

where $a, b$ are positive constants, and $M$ is a nonnegative integer.

1) For $\varrho>0$, if $a b \geq \frac{1}{4}$, then $f=0$ almost everywhere.

2) For $\varrho=0$, we have:

(i) If $a b>\frac{1}{4}$, then $f=0$ almost everywhere.

(ii) If $a b=\frac{1}{4}$, then $f(x)$ is, up to some constant, the heat kernel $h(b, x)$.

Remark 6.4. For $\varrho \geq 0$, if $a b<\frac{1}{4}$, then there exist infinitely many linearly independent functions satisfying $(\mathrm{H1})$ and $(\mathrm{H} 2)$. Indeed, for any $r$ satisfying $b<r<\frac{1}{4 a}$, the heat kernel $h(r, x)$ meets the conditions $(\mathrm{H} 1)$ and $(\mathrm{H} 2)$.

We close this section by a generalization of the Hardy type uncertainty principle, where the uniform conditions are replaced by integrability conditions.

Theorem 6.5 (Cowling-Price's type). Let $f \in L^{2}(\mathbb{R}, A(x) d x)$ and assume that for positive real numbers $a, b$ and nonnegative integers $N_{1}, N_{2}$,

$$
\begin{aligned}
& \int_{\mathbb{R}}\left(\frac{|f(x)| e^{a|x|^{2}} e^{\varrho|x|\left(1-\frac{1}{\tilde{p}}\right)} B(x)^{\frac{1}{2}\left(1-\frac{1}{\tilde{p}}\right)}}{(1+|x|)^{N_{1}}}\right)^{\tilde{p}} A(x) d x<\infty, \\
& \int_{\mathbb{R}}\left(\frac{\left|\mathscr{F}_{A}(f)(\lambda)\right| e^{b|\lambda|^{2}}}{(1+|\lambda|)^{N_{2}}}\right)^{\tilde{q}} \xi(\lambda) d \lambda<\infty,
\end{aligned}
$$

where $1 \leq \tilde{p}, \tilde{q} \leq \infty$.

1) If $a b>\frac{1}{4}$ then $f(x)=0$ almost everywhere.

2) If $a b=\frac{1}{4}$ then

$$
f(x)=\sum_{k=0}^{n} c_{k} \Lambda_{A, x}^{(k)} h(b, x) \quad n<N_{2}-\frac{(2 \alpha+2)}{\tilde{q}},
$$


where $c_{k}$ are complex constants, and the subscript in $\Lambda_{A, x}^{(k)} h(b, x)$ denotes the relevant variable in the $k$-th power of the operator $\Lambda_{A}$ applied to the heat kernel $h(b, x)$. In particular:

(i) If $N_{2} \leq\{(2 \alpha+2) / \tilde{q}\}$ then $f=0$ almost everywhere.

(ii) If $\{(2 \alpha+2) / \tilde{q}\}<N_{2} \leq\{(2 \alpha+2) / \tilde{q}\}+1$ then $f(x)=c_{0} h(b, x)$, with:

(a) For $\varrho>0, c_{0}=0$.

(b) For $\varrho=0, c_{0}$ is an arbitrary constant whenever $N_{1}>\{(2 \alpha+2) / \tilde{p}\}$, otherwise $c_{0}=0$.

Remark 6.6. (1) We pin down that when $\tilde{p}=\tilde{q}=\infty$, the Cowling-Price type theorem implies the Corollary 6.3

(2) For $\varrho \geq 0$, if $a b<\frac{1}{4}$, then, for any $N_{1}, N_{2}$, there exist infinitely many linearly independent functions satisfying (CP1) and (CP2). Indeed, for any $r$ satisfying $b<r<\frac{1}{4 a}$, the heat kernel $h(r, x)$ settles the conditions (CP1) and (CP2) for any $N_{1}, N_{2}$.

Proof of Theorem 6.5. Assume first that $1<\tilde{p}, \tilde{q}<\infty$. Denote by $\tilde{p}^{\prime}$ and $\tilde{q}^{\prime}$ the conjugates of $\tilde{p}$ and $\tilde{q}$, respectively.

Using the condition (CP1) and the asymptotic behavior of $A(x)$ for $|x|$ large, we can choose a suitable $r_{1}>2 \alpha+2$ so that

$$
\begin{aligned}
& \int_{\mathbb{R}} \frac{|f(x)| e^{a|x|^{2}}}{(1+|x|)^{N_{1}+\frac{r_{1}}{\tilde{p}^{\prime}}}} A(x) d x \\
\leq & \left(\int_{\mathbb{R}}\left(\frac{|f(x)| e^{a|x|^{2}} e^{\varrho|x|\left(1-\frac{1}{\tilde{p}}\right)} B(x)^{\frac{1}{2}\left(1-\frac{1}{\tilde{p})}\right)}}{(1+|x|)^{N_{1}}}\right)^{\tilde{p}} A(x) d x\right)^{\frac{1}{\tilde{p}}}\left(\int_{\mathbb{R}} \frac{e^{-\varrho|x|}|x|^{2 \alpha+1} \sqrt{B(x)}}{(1+|x|)^{r_{1}}} d x\right)^{\frac{1}{\tilde{p}^{\prime}}}<\infty .
\end{aligned}
$$

Similarly, by the condition (CP2) and the asymptotic behavior of $\xi(\lambda)$ for $|\lambda|$ large, we can choose a suitable $r_{2}>2 \alpha+2$ so that

$$
\begin{aligned}
\int_{\mathbb{R}} \frac{\left|\mathscr{F}_{A}(f)(\lambda)\right| e^{b|\lambda|^{2}}}{(1+|\lambda|)^{N_{2}+\frac{r_{2}}{\tilde{q}^{\prime}}} \xi(\lambda) d \lambda} & \leq\left(\int_{\mathbb{R}}\left(\frac{\left|\mathscr{F}_{A}(f)(\lambda)\right| e^{b|\lambda|^{2}}}{(1+|\lambda|)^{N_{2}}}\right)^{\tilde{q}} \xi(\lambda) d \lambda\right)^{\frac{1}{\tilde{q}}}\left(\int_{\mathbb{R}} \frac{1}{(1+|\lambda|)^{r_{2}}} \xi(\lambda) d \lambda\right)^{\frac{1}{\tilde{q}^{\prime}}} \\
& <\infty .
\end{aligned}
$$

For $\tilde{p}=1$ and/or $\tilde{q}=1$ the above two inequalities are obvious.

Hence, for $1 \leq \tilde{p}, \tilde{q}<\infty$, the function $f$ satisfies Gelfand-Shilov conditions (GS1) and (GS2) with $p=q=2, a=2 s^{2}, b=2 t^{2}$, and $N=\max \left(N_{1}+\frac{r_{1}}{\tilde{p}^{\prime}}, N_{2}+\frac{r_{2}}{\tilde{q}^{\prime}}\right)$. Notice that the condition $a b \geq 1 / 4$ becomes $s t \geq 1 / 4$.

From Theorem 6.1, we get $f=0$ almost everywhere for $a b>1 / 4$, and when $a b=$ $1 / 4$

$$
f(x)=\sum_{k=0}^{n} c_{k} \Lambda_{A, x}^{(k)} h(b, x) \quad n<N-(2 \alpha+2) .
$$

Moreover, from the proof of Theorem 6.1 we knew that $\mathscr{F}_{A}(f)$ is of the form $\mathscr{F}_{A}(f)(\lambda)=$ $Q(\lambda) e^{-b|\lambda|^{2}}$, where $Q$ is a polynomial of degree $n$. Thus, in view of the condition (CP2), we get the additional condition $n<N_{2}-\{(2 \alpha+2) / \tilde{q}\}$, otherwise $f=0$ almost everywhere 
is the only accommodate function in the inequality (CP2). In conclusion, the integer $n$ in (6.12) should satisfy $n<N_{2}-\{(2 \alpha+2) / \tilde{q}\}$ (which is $<N-(2 \alpha+2)$ ). In particular, if $\{(2 \alpha+2) / \tilde{q}\}<N_{2} \leq\{(2 \alpha+2) / \tilde{q}\}+1$ then $f(x)=c_{0} h(b, x)$ for some $c_{0} \in \mathbb{C}$. By considering the asymptotic expression (6.10) of the heat kernel, it follows that for $\varrho>0$ we should have $c_{0}=0$, otherwise the condition (CP1) does not hold. For $\varrho=0$, using again the asymptotic expression of the heat kernel, we deduce that $h(b, x)$ cannot satisfy the condition (CP1) unless the constant $N_{1}$ in the condition (CP1) verify $N_{1}>$ $\{(2 \alpha+2) / \tilde{p}\}$

Now assume that $\tilde{p}=\infty$ and/or $\tilde{q}=\infty$. Let $g(x):=\frac{|f(x)| e^{a|x|^{2}} e^{\varrho|x|} \sqrt{B(x)}}{(1+|x|)^{N_{1}}}$. The condition (CP1) with $\tilde{p}=\infty$ implies

$$
\int_{\mathbb{R}} \frac{|f(x)| e^{a|x|^{2}}}{(1+|x|)^{N_{1}+r_{1}}} A(x) d x \leq\|g\|_{L_{x}^{\infty}}\left(\int_{\mathbb{R}} \frac{e^{-\varrho|x|}|x|^{2 \alpha+1} \sqrt{B(x)}}{(1+|x|)^{r_{1}}} d x\right)<\infty
$$

whenever $r_{1}>2 \alpha+2$. Similarly, the condition (CP2) with $\tilde{q}=\infty$ implies

$$
\int_{\mathbb{R}} \frac{\left|\mathscr{F}_{A}(f)(\lambda)\right| e^{b|\lambda|^{2}}}{(1+|\lambda|)^{N_{2}+r_{2}}} \xi(\lambda) d \lambda<\infty
$$

whenever $r_{2}>2 \alpha+2$. The statement for $\tilde{p}=\infty$ and/or $\tilde{q}=\infty$ now follows in a similar fashion to what we used above for $1 \leq \tilde{p}, \tilde{q}<\infty$.

\section{REFERENCES}

[1] J.-Ph. Anker, The spherical Fourier transform of rapidly decreasing functions. A simple proof of a characterization due to Harish-Chandra, Helgason, Trombi, and Varadarajan, J. Funct. Anal. 96 (1991), no. 2, 331-349.

[2] S. C. Bagchi and S. K. Ray, Uncertainty principles like Hardy's theorem on some Lie groups, J. Austral. Math. Soc. Ser. A 65 (1998), 289-302.

[3] A. Beurling, The collected works of Arne Beurling, Vol. 2. Harmonic analysis. Edited by L. Carleson, P. Malliavin, J. Neuberger and J. Wermer. Contemporary Mathematicians. Birkhäuser Boston, Inc., Boston, MA, 1989. xx+389 pp.

[4] S. Ben Said, A. Boussen, and M. Sifi, On a family of differential-reflection operators: some aspects of harmonic analysis and applications, preprint (2014).

[5] A. Bonami, B. Demange, and Ph. Jaming, Hermite functions and uncertainty principles for the Fourier and the windowed Fourier transforms, Rev. Mat. Iberoamericana 19 (2003), 23-55.

[6] O. Bracco, Propriétés de la mesure spectrale pour une classe d'opérateurs différentiels singuliers sur $(0, \infty)$, C. R. Acad. Sci. Paris Ser. I Math. 329 (1999), no. 4, 299-302.

[7] __ Fonction maximale associée à des opérateurs de Sturm-Liouville singuliers, Thèse, Université Louis Pasteur (Strasbourg I), Strasbourg, 1999, iv+148 pp.

[8] P. Ciatti, F. Ricci, and M. Sundari, Heisenberg-Pauli-Weyl uncertainty inequalities and polynomial volume growth, Adv. Math. 215 (2007), 616-625.

[9] H. Chébli, Sur un théorème de Paley-Wiener associé à la décomposition spectrale d'un opérateur de Sturm-Liouville sur $(0, \infty)$, J. Functional Analysis 17 (1974), 447-461.

[10] _ Opérateurs de translation généralisée et semi-groupes de convolution, Théorie du potentiel et analyse harmonique (Journées Soc. Math. France, Inst. Recherche Math. Avancée, Strasbourg, 1973), pp. 35-59. Lecture Notes in Math., 404, Springer, Berlin, (1974).

[11] _ Théorème de Paley-Wiener associé à un opérateur différentiel singulier sur $(0, \infty)$, J. Math. Pures Appl. (9) 58 (1979), no. 1, 1-19. 
[12] M. G. Cowling and J. F. Price, Generalizations of Heisenbergs inequality, in: Harmonic Analysis (eds. G. Mauceri, F. Ricci and G. Weiss), LNM, no.992, 443-449, Springer, Berlin, (1983).

[13] C. F. Dunkl, Differential-difference operators associated to reflection groups, Trans. Amer. Math. Soc. 311 (1989), 167-183

[14] W.G. Faris, Inequalities and uncertainty principles, J. Math. Phys. 19 (1978) 461-466.

[15] G.B. Folland and A. Sitaram, The uncertainty principle - a mathematical survey, J. Fourier Anal. Appl. 3 (1997), 207-238.

[16] A. Fitouhi, Heat "polynomials" for a singular differential operator on $(0, \infty)$, Constr. Approx. 5 (1989), 241-270.

[17] I.M. Gelfand and G.E. Shilov, Fourier transforms of rapidly increasing functions and questions of uniqueness of the solution of Cauchy's problem, Uspehi Matem. Nauk 8 (1953), 3-54.

[18] G. H. Hardy, A Theorem Concerning Fourier Transforms, J. London Math. Soc. 8 (1933), 227-231.

[19] V. Havin and B. Jöricke, The uncertainty principle in harmonic analysis, Springer-Verlag, Berlin, 1994.

[20] V. P. Havin, On the uncertainty principle in harmonic analysis, Twentieth century harmonic analysis-a celebration, (Il Ciocco, 2000, Italy), NATO Sci. Ser. II Math. Phys. Chem 33, 2001.

[21] G. J. Heckman, Dunkl operators, Séminaire Bourbaki, Vol. 1996/97. Astérisque No. 245 (1997), Exp. No. 828, 4, 223-246.

[22] L. Hörmander, A uniqueness theorem of Beurling for Fourier transform pairs, Ark. Mat. 29 (1991), 237-240

[23] S. Hamem and L. Kamoun, Uncertainty principle inequalities related to Laguerre-Bessel transform, Math. Inequal. Appl. 16 (2013), no. 2, 375-387.

[24] R. Ma, Heisenberg uncertainty principle on Chébli-Trimèche hypergroups, Pacific J. Math. 235 (2008), no. 2, 289-296.

[25] G. W. Morgan, A note on Fourier transforms, J. London Math. Soc. 9 (1934), 187-192.

[26] M. A. Mourou and K. Trimèche, Transmutation operator and Paley-Wiener theorem associated with a singular Differential-difference operator on the real line, Anal. Appl. 1 (2003), pp. 43-70.

[27] E. K. Narayanan and S. K. Ray, The heat kernel and Hardy's theorem on symmetric spaces of noncompact type, Proc. Indian Acad. Sci. Math. Sci. 112 (2002), 321-330.

[28] S. Omri and L. T. Rachdi, Heisenberg-Pauli-Weyl uncertainty principle for the Riemann-Liouville operator, J. Inequal. Pure Appl. Math. 9, 3 (2008), 1-23.

[29] J. F. Price, Inequalities and local uncertainty principles, J. Math. Phys 24 (1983), 1711-1714.

[30] Sharp local uncertainty principles, Studia Math. 85 (1987) 37-45.

[31] E. R. P. Sarkar and J. Sengupta, Beurling's theorem and characterization of heat kernel for Riemannian symmetric spaces of noncompact type, Canad. Math. Bull. 50 (2007), 291-312.

[32] A. Sitaram and M. Sundari, An analogue of Hardy's theorem for very rapidly decreasing functions on semi-simple Lie groups, Pacific J. Math. 177 (1997), 187-200.

[33] S. Thangavelu, On theorems of Hardy, Gelfand-Shilov and Beurling for semisimple groups, Publ. Res. Inst. Math. Sci. 40 (2004), 311-344.

[34] K. Trimèche, The transmutation operators relating to a Dunkl type operator on $\mathbb{R}$ and their positivity, Mediterr. J. Math. (2014), to appear.

[35] _ Transformation intégrale de Weyl et théorème de Paley-Wiener associés à un opérateur difftrentiel singulier sur (0, $)$, J. Math. Pures Appl. (9) 60 (1981), no. 1, 51-98.

[36] _ Generalized wavelets and hypergroups, Gordon and Breach Science Publishers, Amsterdam, 1997. xii+354 pp.

[37] O. L. Vinogradov, On the norms of generalized shift operators generated by Dunkl-type operators, J. Math. Sci. (N. Y.) 184 (2012), no. 6, 663-678

[38] H. Weyl, Gruppentheorie und Quantenmechanik, S. Hirzel, Leipzig, 1928. Revised English edition: The Theory of Groups and Quantum Mechanics, Methuen, London, 1931; reprinted by Dover, New York, 1950. 
S. Ben Said: Institut Élie Cartan, Université de Lorraine-Nancy, B.P. 239, F-54506 VandoeuvresLÈs-NANCY, FRANCE

E-mail address: salem.bensaid@univ-lorraine.fr

A. Boussen et M. Sifi: Université de Tunis El Manar, Faculté des Sciences de Tunis, LR11ES11 Laboratoire d’Analyse Mathématiques et Applications, 2092, Tunis, Tunisie

E-mail address: asma.boussen@live.fr, mohamed.sifi@fst.rnu.tn 